\title{
Electromagnetic design of superconducting quadrupoles
}

\author{
L. Rossi and E. Todesco \\ Accelerator Technology Department, CERN, CH 1211, Geneva
}

(Received 19 March 2006; published 12 October 2006)

\begin{abstract}
We study how the critical gradient depends on the coil layout in a superconducting quadrupole for particle accelerators. We show that the results relative to a simple sector coil are well representative of the coil layouts that have been used to build several quadrupoles in the past 30 years. Using a semianalytical approach, we derive a formula that gives the critical gradient as a function of the coil cross-sectional area, of the magnet aperture, and of the superconducting cable parameters. This formula is used to evaluate the efficiency of several types of coil layouts (shell, racetrack, block, open midplane).
\end{abstract}

DOI: $10.1103 /$ PhysRevSTAB.9.102401

PACS numbers: 07.55.Db, 41.85.Lc, 84.71.Ba, 85.70.Ay

\section{INTRODUCTION}

Superconducting quadrupoles are widely used to focus particle beams in accelerator machines. The technology based on $\mathrm{Nb}-\mathrm{Ti}$ is mature, having been used for 30 years [1-3] in high energy physics machines such as the Intersecting Storage Ring (ISR) [4], the Tevatron [5], the Hadron Elektron Ring Anlage (HERA) [6], the Large Electron Positron (LEP) [7,8], the ill-fated Colliding Beam Accelerator (CBA) [9] and Superconducting Super Collider (SSC) [10], the Relativistic Heavy Ion Collider (RHIC) [11], and the Large Hadron Collider (LHC) now under construction [12]. The strongest motivation for pursuing quadrupoles with higher gradients and larger apertures is the future upgrade of the interaction regions of the LHC, where the aim is the reduction of the beam size in the collision point to obtain higher peak luminosity [13-15]. However, other projects, such as the International Linear Collider (ILC) [16], might profit from a quadrupole development, in the case that wide low beta quadrupoles are needed for the interaction point. This pushes the community to use materials with better superconducting properties, such as the $\mathrm{Nb}_{3} \mathrm{Sn}$ [17-22].

The electromagnetic design of the coil layout of a superconducting quadrupole has to take into account two main aspects: the peak field in the coil must stay below the limit of the superconducting material, and the field shape has to be very close to a pure quadrupolar term, i.e., within $10^{-4}$. In the past, several options for the coil layout have been adopted: (i) different geometry of cables in terms of width, thickness and keystone angle; (ii) different numbers of layers of conductors (one, two, or four) to get the highest gradient; (iii) different numbers and arrangement of spacers or wedges to optimize the field quality.

Most of the accelerator quadrupoles have been built using the so-called shell (or $\cos 2 \theta$ ) design, where the conductor is arranged in sectors centered around the magnet aperture. Designs with a simpler geometry (block coil [17] or racetrack coil [20,21]) have been proposed and successfully built in small models, but it is believed that they are intrinsically less efficient in their use of superconducting material.

At the very early stage of the development of superconducting magnets, the design was only guided by analytical tools $[1,23]$. Then, computer codes that numerically solve the equations for a given coil design have been made available $[24,25]$, enabling the comparison of several design options to find the best solution. Indeed, since the parameter space is very large, one cannot be sure that the obtained solution is the optimum one. Moreover, the starting point of the optimization process needs either an analytical insight or what is usually defined as "experience." A systematic comparison of the design choices in terms of their parametric dependence on the magnet aperture and needed gradient is still not available in the literature.

The issues we address in this paper are the following ones: (i) estimating the highest gradient that can be obtained for a given aperture radius; (ii) finding the coil layout that produces the highest field gradient for a given aperture, independently of the quantity of superconductor (neither cost nor size constraints); (iii) comparing the different coil layouts when seeking the highest field gradient reachable with a given quantity of superconductor; (iv) obtaining an analytical approximation for the field gradient as a function of the coil dimension, geometry and of the superconducting properties. This law can be used to give a cost estimate (based on the quantity of the superconducting cable) and a guess of the magnet size (based on the dimension of the coil) for a given aperture and required field gradient.

This analysis is focused on the electromagnetic design. Therefore, we ignore all aspects related to the magnet protection, to the mechanical structure needed to withstand the electromagnetic forces [26-28], to the effects of the mechanical stresses on the conductor performance, and to the superconductor stability. The results are mainly given for the case of $\mathrm{Nb}-\mathrm{Ti}$, but the approach can be generalized to other materials. 
As in Refs. [27-29], we focused on simplified coil models, relying when possible on analytical tools to have a theoretical insight. For quantities that we could not compute analytically, we used a dedicated numerical code [30], carrying out an exploration of the parameter space, and making analytical fits to the numerical results.

We mainly focus our analysis to the shell design, and we outline some estimates for other geometries. Since the results are given in terms of the cross-sectional conductor area (insulation included), they can be used as a benchmark to evaluate the efficiency of all types of coil layouts. We finally compare the obtained results to the actual values of $13 \mathrm{Nb}$-Ti quadrupoles that have been built and tested during the past 30 years.

In Sec. II we introduce the equations that define the field gradient in terms of the properties of the superconductor, of the coil design, and of the aperture radius. A simple model that can be completely solved in an analytical way is presented in Sec. III. Scaling laws to extrapolate the results found for a given aperture radius to all apertures are derived in Sec. IV. Several cases of the shell layout are analyzed in Sec. V, and other types of designs are outlined in Sec. VI. The comparison of the obtained scaling laws to the data relative to 13 quadrupoles designs that have been built for accelerators is given in Sec. VII. The impact of the iron on our analysis is discussed in Sec. VIII, and an application of the scaling laws to the LHC insertion upgrade is outlined in Sec. IX. Hints on the $\mathrm{Nb}_{3} \mathrm{Sn}$ limits are given in Sec. X. A summary is given in Sec. XI, and technical details about field quality and explicit computations are grouped in the Appendices.

\section{EQUATIONS DEFINING THE LIMIT GRADIENT}

\section{A. Critical current density}

Let us consider a $\mathrm{Nb}$-Ti filament carrying a current density $j_{\mathrm{sc}}$ in a magnetic field $B$. The filament is superconducting as long as the current density is less than the critical current density $j_{\mathrm{sc}, c}$, which can be approximated by a linear function of the magnetic field:

$$
j_{\mathrm{sc}, c}=c\left(B_{c 2}^{*}-B\right)
$$

where (i) $B^{*}{ }_{c 2}[\mathrm{~T}]$ is the critical field at zero current according to the linear fit, that underestimates the actual critical field value of around $10 \%$ (see Fig. 1). (ii) $c$ $\left[\mathrm{A} /\left(\mathrm{T} \mathrm{m}^{2}\right)\right]$ is the slope of the line in the $\left(j_{\mathrm{sc}}, B\right)$ plane.

The fit is good for values of the magnetic field larger than $5 \mathrm{~T}$ at $1.9 \mathrm{~K}$, and $2 \mathrm{~T}$ at $4.2 \mathrm{~K}$, which is the interesting domain for our analysis. For the Nb-Ti, $B^{*}{ }_{c 2} \sim 10 \mathrm{~T}$ at $4.2 \mathrm{~K}$ and $\sim 13 \mathrm{~T}$ at $1.9 \mathrm{~K}$, whereas the slope $c \sim 6 \times$ $10^{8} \mathrm{~A} /\left(\mathrm{T} \mathrm{m}^{2}\right)$ is independent of the temperature. This corresponds to having $3 \times 10^{9} \mathrm{~A} / \mathrm{m}^{2}$ (i.e. $3000 \mathrm{~A} / \mathrm{mm}^{2}$ ) at $8 \mathrm{~T}$ and $1.9 \mathrm{~K}$, or at $5 \mathrm{~T}$ and $4.2 \mathrm{~K}$. Please note that throughout the paper lengths will be expressed in meters in

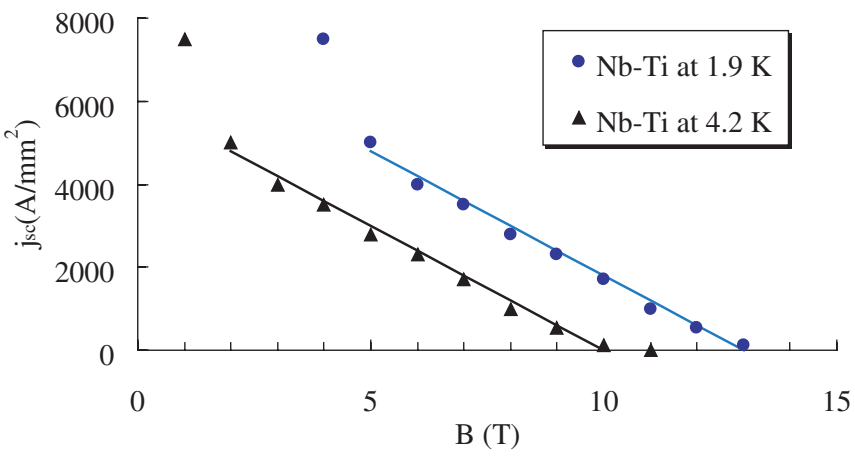

FIG. 1. (Color) Critical surface for $\mathrm{Nb}-\mathrm{Ti}$ at $1.9 \mathrm{~K}$ and at $4.2 \mathrm{~K}$, and linear fit given in Eq. (1).

all equations and constants, whereas in some figures and tables millimeters will be used to improve the readability (for instance, current density is shown in $\mathrm{A} / \mathrm{mm}^{2}$ in Figs. 1 and 3).

A practical superconductor wire is made of $\mathrm{Nb}$-Ti filaments in a copper matrix, and one defines (iii) $\nu_{\mathrm{Cu}-\mathrm{sc}}$ (dimensionless) as the copper to superconductor ratio, i.e., the ratio between the quantity of copper and the quantity of superconductor in the strands. Its value is ranging from 1 to 2 for typical high current density conductors.

The wires are then assembled in cables, to obtain conductors with high operating currents, and finally insulated. These steps bring an additional dilution of the quantity of superconductor present in the section of the insulated cable ready for winding, which can be estimated in 10\%-15\% for each step. We define $\kappa_{w-c}$ as the ratio between the area of the strands in the conductor and the area of the bare conductor, and $\kappa_{c-i}$ as the ratio between the area of the bare conductor and of the insulated conductor. The current density $j$ flowing in the insulated conductor is therefore given by

$$
j=\kappa_{w-c} \kappa_{c-i} \frac{j_{s c}}{1+\nu_{\mathrm{Cu}-\mathrm{sc}}}=\kappa j_{\mathrm{sc}}
$$

where we defined the filling factor $\kappa$

$$
\kappa=\kappa_{w-c} \kappa_{c-i} \frac{1}{1+\nu_{\mathrm{Cu}-\mathrm{sc}}} .
$$

The linear fit for the critical current surface at a given temperature can then be written as

$$
j_{c}=\kappa c\left(B_{c 2}^{*}-B\right)
$$

\section{B. Critical gradient, current, and peak field}

We now consider a quadrupole coil cross section, i.e., a layout of conductors that satisfies a fourfold symmetry and 


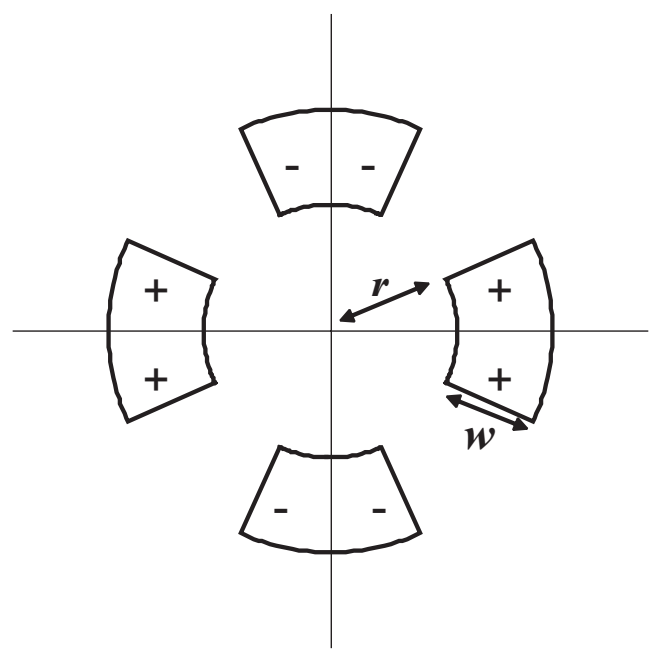

FIG. 2. Layout of a $30^{\circ}$ sector coil for a quadrupole of aperture radius $r$ and coil width $w$.

where the current is flowing in opposite directions in each adjacent coil (see Fig. 2, where a $30^{\circ}$ sector coil is shown).

We assume that the magnetic field is entirely given by the current lines, i.e., that there is no contribution given by the iron, and that the current density $j$ in the coil is uniform. The current density is defined as the conductor current divided by the cross-sectional surface of the insulated conductor. We then define: (i) the field gradient $G[\mathrm{~T} / \mathrm{m}]$ at the center of the quadrupole; (ii) the peak field $B_{p}[\mathrm{~T}]$, i.e., the largest value (in module) of the magnetic field in the coil. One can prove that, for uniform $j$, the field is maximum on the contour line of the coils [29].

Because of the linearity of the Biot-Savart law, both $G$ and $B_{p}$ are proportional to the current density in the coil $j$ :

$$
\begin{gathered}
G=j \gamma, \\
B_{p}=j \beta,
\end{gathered}
$$

where we define the following parameters that characterize the coil layout: (i) $\gamma[\mathrm{T} \mathrm{m} / \mathrm{A}]$ is the field gradient (in $\mathrm{T} / \mathrm{m}$ ) per unit of current density (in $\mathrm{A} / \mathrm{m}^{2}$ ); (ii) $\beta\left[\mathrm{T} \mathrm{m}^{2} / \mathrm{A}\right]$ is the peak field (in T) per unit of current density (in $\mathrm{A} / \mathrm{m}^{2}$ ).

Substituting Eq. (4) in Eq. (6) we obtain

$$
B_{p, c}=j_{c} \beta=\kappa c\left(B_{c 2}^{*}-B_{p, c}\right) \beta
$$

and we can solve for the critical peak field $B_{p, c}$, that is reached in the coil when the critical surface is hit (see Fig. 3):

$$
B_{p, c}=\frac{\kappa c B_{c 2}^{*}}{1+\kappa c \beta} \beta
$$

This corresponds to a critical current $j_{p, c}$

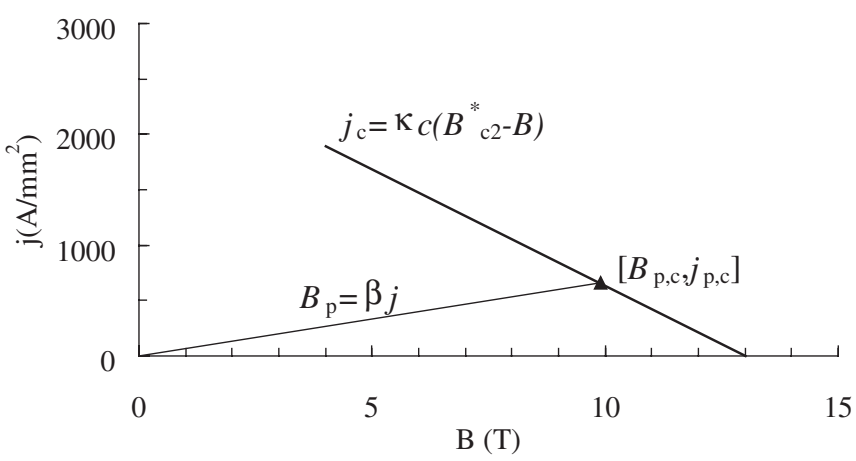

FIG. 3. Example of critical surface, load line, critical current, and critical peak field for the LHC main quadrupole (1.9 K).

$$
j_{p, c}=\frac{\kappa c B_{c 2}^{*}}{1+\kappa c \beta}
$$

that gives the critical gradient

$$
G_{c}=\frac{\kappa c B_{c 2}^{*}}{1+\kappa c \beta} \gamma
$$

which is sometimes improperly called quench gradient. As the quench is also determined by the mechanical stability and by the cooling conditions of the cable, the denomination of critical gradient is more appropriate.

\section{Field limited and current limited regimes}

Both $\gamma$ and $\beta$ defined in (5) and (6) are dependent on the quantity of conductor and on the coil shape. If a very little quantity of conductor is used, $\gamma$ and $\beta$ are "small," and they increase when more conductor is added to the coil layout. The previous formulas (8)-(10) suggest that there are two distinct regimes.

(i) $\kappa c \beta \ll 1$. - In this case the critical current is equal to $\kappa c B^{*}{ }_{c 2}$, i.e., it depends only on the superconducting properties and it is independent of the coil layout. Moreover, the critical gradient is equal to $\kappa c \gamma B^{*}{ }_{c 2}$ and independent of $\beta$. When we add more cable, the corresponding increase of $\gamma$ directly affects the critical gradient. An increase of the filling ratio $\kappa$ also directly affects the critical gradient. We will denote this regime as current limited.

$\kappa c \beta \gg 1$.- In this case the critical peak field tends to $B^{*}{ }_{c 2}$, and the critical current tends to zero. All quantities become independent of $\kappa$. The critical gradient is

$$
G_{c} \approx B_{c 2}^{*} \frac{\gamma}{\beta}
$$

and the interplay between the increase of $\gamma$ and $\beta$ for larger and larger coils determines the maximum critical gradient. We will denote this regime as field limited.

In Table I we compute this factor for 13 magnets designs that have been manufactured in the past 30 years. The 
TABLE I. Factor $\kappa c \beta$ for some superconducting magnets.

\begin{tabular}{lcccccc}
\hline \hline Name & $\begin{array}{c}\text { Aperture radius } \\
(\mathrm{mm})\end{array}$ & $\begin{array}{c}\nu_{\mathrm{Cu} / \mathrm{sc}} \\
\text { (adimensional) }\end{array}$ & $\begin{array}{c}\kappa \\
(\text { adimensional) }\end{array}$ & $\begin{array}{c}c \\
\mathrm{~A} /\left(\mathrm{T} \mathrm{m}^{2}\right)\end{array}$ & $\begin{array}{c}\beta \\
\mathrm{T} \mathrm{m}^{2} / \mathrm{A}\end{array}$ & $\begin{array}{c}\kappa c \beta \\
\text { (adimensional) }\end{array}$ \\
\hline ISR MQ & 116 & 1.45 & 0.35 & $6.00 \times 10^{+08}$ & $2.06 \times 10^{-08}$ & 4.3 \\
Tevatron MQ & 45 & 1.85 & 0.25 & $6.00 \times 10^{+08}$ & $1.02 \times 10^{-08}$ & 1.5 \\
HERA MQ & 37 & 1.80 & 0.27 & $6.00 \times 10^{+08}$ & $1.12 \times 10^{-08}$ & 1.8 \\
SSC MQ & 20 & 1.78 & 0.27 & $6.00 \times 10^{+08}$ & $9.31 \times 10^{-09}$ & 1.5 \\
LEP I MQC & 90 & 1.70 & 0.31 & $6.00 \times 10^{+08}$ & $1.77 \times 10^{-08}$ & 3.3 \\
LEP II MQC & 80 & 1.60 & 0.33 & $6.00 \times 10^{+08}$ & $1.49 \times 10^{-08}$ & 3.0 \\
RHIC MQ & 40 & 2.25 & 0.23 & $6.00 \times 10^{+08}$ & $6.71 \times 10^{-09}$ & 0.9 \\
RHIC MQY & 65 & 1.80 & 0.27 & $6.00 \times 10^{+08}$ & $8.64 \times 10^{-09}$ & 1.4 \\
LHC MQ & 28 & 1.95 & 0.25 & $6.00 \times 10^{+08}$ & $1.41 \times 10^{-08}$ & 2.1 \\
LHC MQM & 28 & 1.75 & 0.26 & $6.00 \times 10^{+08}$ & $9.80 \times 10^{-09}$ & 1.5 \\
LHC MQY & 35 & 1.25 & 0.34 & $6.00 \times 10^{+08}$ & $1.88 \times 10^{-08}$ & 3.9 \\
LHC MQXA & 35 & 1.20 & 0.34 & $6.00 \times 10^{+08}$ & $1.99 \times 10^{-08}$ & 4.1 \\
LHC MQXB & 35 & 1.30 & 0.33 & $6.00 \times 10^{+08}$ & $1.60 \times 10^{-08}$ & 3.1 \\
\hline \hline
\end{tabular}

copper to superconductor ratio can vary from 1.2 to 2.2 , and the additional effect of interstrand voids and insulation gives a dilution factor $\kappa$ that ranges from 0.23 to 0.35 . Please note that for magnets with current grading (last three in Table I), we give values relative to the cables that limit the critical gradient (i.e., the inner layer in all cases). Since the aim of this work is to compare the electromagnetic design and not the performance of the superconductor, for all magnets we set the slope $c$ of the critical surface to the same value of $6 \times 10^{8} \mathrm{~A} /\left(\mathrm{T} \mathrm{m}^{2}\right)$.

Most of the magnets listed in Table I are in an intermediate regime where $\kappa c \beta$ is of the order of 1 . Magnets with a large amount of superconductor such as the LHC MQXA, MQXB, and MQY, and with a very large aperture radius (ISR and LEP) are close to the field limited regime.

\section{Towards the highest critical gradients}

A simple heuristic argument can be used to define the theoretical maximum critical gradient for a magnet of aperture $r$ and superconducting material with a critical field at $B_{c 2}^{*}$. For an ideal quadrupole, the field on the coil inner radius $r$ is equal to $r G$, and therefore the peak field on the coil satisfies

$$
B_{p, c} \geq r G_{c} .
$$

Since the peak field must always be smaller than the maximum field of the material $B^{*}{ }_{c 2}$, one has that

$$
G_{c} \leq \frac{B_{p, c}}{r} \leq \frac{B_{c 2}^{*}}{r} \equiv G_{c}^{*} .
$$

For instance for $\mathrm{Nb}-\mathrm{Ti}$ at $1.9 \mathrm{~K}$, one has $B^{*}{ }_{c 2}=13 \mathrm{~T}$ and therefore the critical gradient can never be larger than $13 / r$, i.e. $G_{c}{ }^{*}=433 \mathrm{~T} / \mathrm{m}$ for an aperture radius of $30 \mathrm{~mm}$ or $G_{c}{ }^{*}=289 \mathrm{~T} / \mathrm{m}$ for an aperture radius of $45 \mathrm{~mm}$ (see Fig. 4).

Equation (10) shows that, to increase the critical gradient, one can act on three separate aspects. (i) Improve the superconducting parameters of the conductor: the straightforward way is of choosing a material with larger critical field $B^{*}{ }_{c 2}$. The increase of the slope $c$ also gives a higher critical gradient, but this action becomes less and less effective when $\kappa c \beta \gg 1$.

(ii) Improve the quantity of superconductor in the conductor, i.e., increase $\kappa$. This action becomes less and less effective when we approach the field limited regime $\kappa c \beta \gg 1$.

(iii) Improve the parameters of the coil layout, namely, increase $\gamma$ and reduce $\beta$. These two objectives are obviously competing, since when we use more conductor we increase both $\gamma$ and $\beta$. In the current limited regime, the improvements of $\gamma$ directly affect $G_{c}$. On the other hand, in the field limited regime the increase of $\gamma$ can be either totally compensated or even over-ruled by the increase of $\beta$, without giving any beneficial effect on $G_{c}$. The interplay between the dependence of $\gamma$ and $\beta$ on the coil layout constitutes the problem of the optimization of the coil cross section.

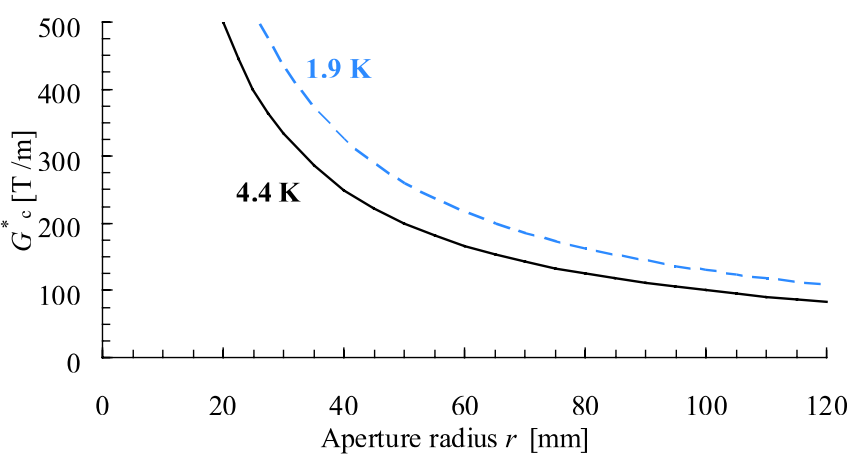

FIG. 4. (Color) Theoretical upper bound $G^{*}{ }_{c}$ to critical gradient for $\mathrm{Nb}-\mathrm{Ti}$ as a function of the aperture radius. 


\section{A SIMPLE MODEL: THE CROSS QUADRUPOLE}

\section{A. Aim of the model and coil layout}

To demonstrate the principle of our approach, we start by analyzing a simple model where explicit expressions for $\gamma$ and $\beta$ in terms of the geometrical quantities of the design can be derived. We consider a quadrupole coil layout as sketched in Fig. 5, where one octant of the coil is made up of a rectangular conductor of width $w$ and thickness $t / 2$ placed on the coordinate axis at a distance $r$ from the center. We will show that this model presents features that are common to more realistic coil layouts of superconducting quadrupoles. In the model used for numerical computation, we fix the thickness at $1 \mathrm{~mm}$.

\section{B. Evaluation of the field gradient}

The field gradient can be computed using the BiotSavart law (see Appendices A and B for details), and is proportional to the integral of the inverse of the square of the distance $\rho$ from the center [see Eq. (A7)]

$$
G \propto \int_{r}^{r+w} \frac{d \rho}{\rho^{2}}=\frac{1}{r+w}-\frac{1}{r}=-\frac{w}{r(r+w)} .
$$

The evaluation of the exact expression is given in (B1):

$$
G=\frac{8}{5} t \frac{w}{r(r+w)} j
$$

and therefore the parameter $\gamma$ [see Eq. (5)] is

$$
\frac{G}{j}=\gamma(r, w)=\frac{8}{5} t \frac{w}{r(r+w)} .
$$

For $w \ll r$, we have the current limited regime and $\gamma$ is proportional to $w$

$$
\gamma \propto \frac{w}{r^{2}} .
$$

For $w \gg r$, we have the field limited regime and $\gamma$ satu-

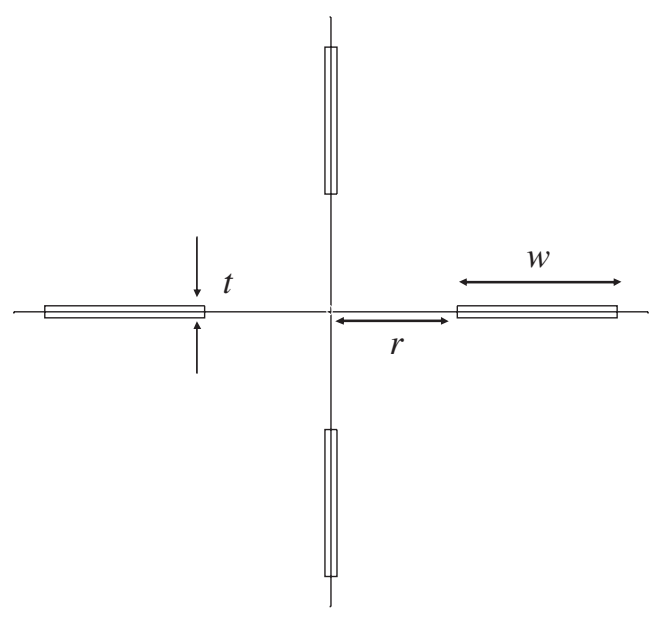

FIG. 5. Coil layout of the cross quadrupole. rates:

$$
\gamma \propto \frac{8}{5} \frac{t}{r},
$$

i.e. increasing the cable width $w$ does not improve $\gamma$.

\section{Evaluation of the peak field}

The peak field in the coil is computed using the BiotSavart law and is proportional to the integral of the inverse of the distance. The details of the analytical computations are given in Appendix B, Eqs. (B2)-(B10). One can prove that for small and medium $w / r$ the peak field is on the inner side of the conductor, facing the center of the aperture, whereas for large $w / r$ the peak field is on the outer side of the conductor. This second case is far from the usual domain of interest for superconducting magnets.

For all $w$, one can approximate the peak field (within $10 \%$ ) by neglecting the contribution of the three conductors that are far from the location of the peak field [see Eq. (B10)]. Therefore one can write

$$
B_{p}=\beta j \sim\left[\beta_{0}+\beta_{1} \log \left(\frac{w}{w_{0}}\right)\right] j,
$$

where $\beta_{1}=4 \times 10^{-10}$, and $\beta_{0}$ is related to the constant $w_{0}$ used to remove the logarithmic singularity due to the fact that we are considering a sheet of current with $t \ll 1$. In this approximation, $\beta$ is independent of the aperture radius $r$.

\section{Evaluation of the critical gradient}

Using Eqs. (9) and (19), one obtains an approximated expression for the critical current in the cross quadrupole,

$$
j_{p, c}(w) \sim \frac{\kappa c B_{c 2}^{*}}{1+\kappa c\left[\beta_{0}+\beta_{1} \log \left(w / w_{0}\right)\right]} .
$$

For large values of $w$, the critical current density tends to zero as $1 / \log (w)$. The critical gradient is given by (10), (16), and (19)

$$
G_{c}(r, w) \sim \frac{8}{5} t \frac{w}{r(r+w)} \frac{\kappa c B_{c 2}^{*}}{1+\kappa c\left[\beta_{0}+\beta_{1} \log \left(w / w_{0}\right)\right]} .
$$

The dependence of $G_{c}$ on the cable width $w$ for different values of the aperture radius $r$ is given in Fig. 6. Here, the results of the numerical code are compared to the analytical formula (21) for a $\mathrm{Nb}-\mathrm{Ti}$ conductor at $1.9 \mathrm{~K}$ with a dilution factor [see Eq. (3)] $\kappa=0.33$. The agreement in the analyzed range (apertures from 10 to $70 \mathrm{~mm}$, and cable width from 2 to $1000 \mathrm{~mm}$ ) is within $4 \%$. The only approximation of the analytical model is the estimate of $\beta$ given in Eq. (19).

The rather complex equation (21) has the following features. 


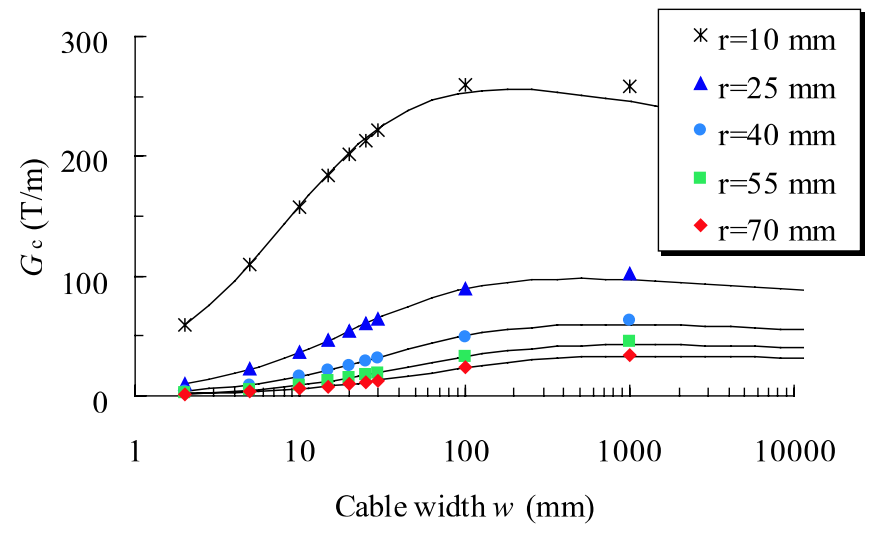

FIG. 6. (Color) Critical gradient versus cable width for different apertures: numerical results (dots) and analytical approximation (solid lines) for the cross-quadrupole model, for $\kappa=0.33$.

(i) For small $w$, the critical gradient is proportional to $w$, i.e., when the cable is very small, a relative increase of the cable dimension brings to the same relative increase of the critical gradient.

(ii) For large $w$, the factor at the denominator $(r+w)$ becomes relevant and $G_{c}$ starts to saturate. A large increase of the cable width leads to only a marginal gain in the gradient.

(iii) For very large $w$, the factor with the logarithm becomes dominant, and $G_{c}$, after having reached a maximum value, tends to zero with the inverse logarithm of the cable width. This is due to the fact that for very large $w$, the field gradient per current density $\gamma$ saturates [see Eqs. (16) and (18)], whereas the peak field per current density continues to increase with $\log (w)$ [see Eq. (19)]. Therefore, when adding more conductor we only increase the peak field (and therefore decrease the critical density current), thus decreasing the critical gradient (see Fig. 6).

We conclude that there is an optimum cable width that provides the maximum critical gradient. In the following section, we will prove that this feature is found also for some realistic layouts based on sector coils. The maximum critical gradient for the cross quadrupole is found to be between $20 \%$ and $25 \%$ of $G_{c}{ }^{*}$ as defined in Eq. (13), with a weak dependence on the aperture radius $r$ and on the dilution factor $\kappa$.

\section{EFFECT OF SPACE RESCALING}

In this section we derive the behavior of the parameters $\gamma$ and $\beta$ in the presence of a rescaling of the space. The aim of this analysis is to extend the results obtained for a given $r$ to all aperture radii in an analytical way, i.e., without the need of numerical simulations. We consider a coil layout characterized by a given shape and by two parameters, namely, the aperture radius $r$ and the coil width $w$ as the sector coil shown in Fig. 2. We then apply a rescaling to the coil cross section, namely $r^{\prime}=\alpha r$ and $w^{\prime}=\alpha w$. (i) The parameter $\gamma$ is proportional to the integral of a surface element divided by the square of a distance [see Eq. (A7)], and therefore is invariant under a space rescaling:

$$
\gamma(\alpha r, \alpha w)=\gamma(r, w)
$$

(ii) The parameter $\beta$ is proportional to the integral of a surface element divided by a distance, and therefore $\beta / r$ is invariant under a space rescaling:

$$
\beta(\alpha r, \alpha w)=\alpha \beta(r, w) .
$$

It should be noted that we are dealing with the case of a two-dimensional coil. In the nonphysical hypothesis of a one-dimensional coil (such as the cross quadrupole discussed in the previous section), the integral is not over a surface element but over a line, and therefore one has $\gamma(\alpha r, \alpha w)=\gamma(r, w) / \alpha$ and $\beta(\alpha r, a w)=\beta(r, w)$, in agreement with the expressions derived in the previous section.

Substituting (22) and (23) in the definition of the critical gradient (10), one can compute the effect of a space rescaling:

$$
G_{c}(\alpha r, \alpha w)=\frac{\kappa c B_{c 2}^{*} \gamma(\alpha r, \alpha w)}{1+\kappa c \beta(\alpha r, \alpha w)}=\frac{\kappa c B_{c 2}^{*} \gamma(r, w)}{1+\alpha \kappa c \beta(r, w)} .
$$

This proves that a rescale of the space does not induce a simple rescaling of the critical gradient. This result is very important since it implies that the problem of the electromagnetic optimization of quadrupoles with "small" or "large" apertures can be radically different. Once again, we have two different regimes.

(i) For the current limited regime $k c \beta \ll 1$, a magnification of the space by a factor $\alpha$ gives the same critical gradient.

(ii) For the field limited regime $k c \beta \gg 1$, a magnification of the space by a factor $\alpha$ reduces the critical gradient by a factor $\alpha$. For instance, a magnet with the same coil shape but with double the aperture and double the coil size will have in this regime half the gradient. This is due to the fact that we are limited by the critical field $B^{*}{ }_{c 2}$ on the coil, and therefore the product of the field gradient and of the aperture radius is a constant.

\section{ANALYSIS OF THE CIRCULAR SHELL (SECTOR) LAYOUT}

\section{A. Layout description}

We first consider a shell design composed by one sector of radial width $w$, of $30^{\circ}$ azimuthal width, at a distance $r$ from the aperture center (see Fig. 2). This well-known textbook example sets to zero the first order field harmonic $b_{6}$ (see Appendix C). 
In order to reach the field quality necessary for an accelerator magnet, a wedge can be put in the sector design: a typical solution is to have a sector of superconductor from $0^{\circ}$ to $24^{\circ}$, a wedge from $24^{\circ}$ to $30^{\circ}$, and a second sector of superconductor from $30^{\circ}$ to $36^{\circ}$. In this way one can set $b_{6}=b_{10}=0$. Indeed, there is a whole one-parameter family of solutions of two-sector layouts that set $b_{6}=b_{10}=0$. The optimization of the first two harmonics is giving the required field homogeneity for accelerators (we recall that codes for tracking the particle motion are usually modeling magnetic fields up to order 11 , and therefore a $b_{14}$ component would be neglected). A detailed analysis is given in Appendix C. Here we will analyze the solutions $\left[0^{\circ}-24^{\circ}, 30^{\circ}-36^{\circ}\right]$ and $\left[0^{\circ}-\right.$ $18^{\circ}, 22^{\circ}-32^{\circ}$ ] shown in Fig. 7.

In several magnets, two or four layers of cable are used. Neglecting the issue of using different $j$ in the two layers, we observe that in some cases the layers have very similar pole angles (for instance, the LHC MQ; see Fig. 8, left). These coil layouts are equivalent to a single shell with a double width. In other cases, the pole angles of the layers differ considerably (for instance, the LHC MQY; see Fig. 8, right). This second layout cannot be approximated by a single shell. In Appendix $\mathrm{C}$ we treat the field quality constraints for a two-layer, one- $j$ configuration, where each layer has the same thickness $w$, showing that if $w / r<$ 0.16 there is a coil layout without copper wedges that sets $b_{6}=b_{10}=0$ [see Eqs. (C8) and (C9), and Fig. 9]. For $w / r>0.16$ we extend this solution by fixing the inner layer angle to $35^{\circ}$ and the outer layer angle at $12.5^{\circ}$, and opening the space for a copper wedge around $24^{\circ}$. One can then obtain a solution that sets $b_{6}=b_{10}=0$ for all cable thicknesses, and that for very large $w$ tends to the solution that one has for a single layer of $35^{\circ}$ and with a copper wedge.

Summarizing, these are the four cases of the shell design we are going to study: (i) the $\left[0^{\circ}-30^{\circ}\right]$ sector (one layer, no copper wedge, $b_{6}=0$ ); (ii) the $\left[0^{\circ}-24^{\circ}, 30^{\circ}-36^{\circ}\right]$ sectors (one layer of $36^{\circ}$ angular width, two sectors, wedge between $24^{\circ}$ and $30^{\circ}, b_{6}=b_{10}=0$ ); (iii) the $\left[0^{\circ}-\right.$ $18^{\circ}, 22^{\circ}-32^{\circ}$ ] sectors (one layer of $32^{\circ}$ angular width,
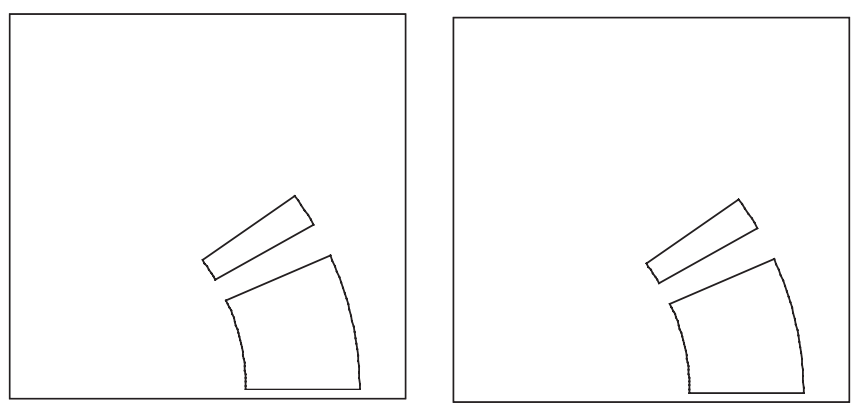

FIG. 7. Two-sector layouts (one eight shown in the plot) $\left[0^{\circ}-\right.$ $\left.24^{\circ}, 30^{\circ}-36^{\circ}\right]$ (left) and $\left[0^{\circ}-18^{\circ}, 22^{\circ}-32^{\circ}\right]$ (right) that set $b_{6}$ and $b_{10}=0$.
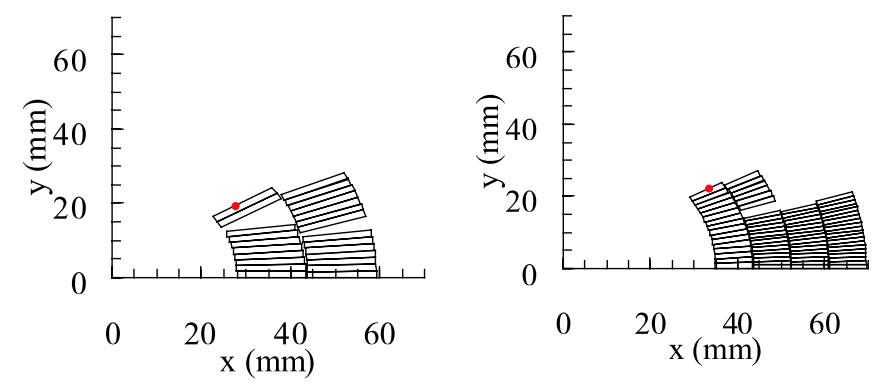

FIG. 8. (Color) Coil layouts (one eight shown in the plot) of the LHC MQ (left) and MQY (right).

two sectors, wedge between $18^{\circ}$ and $22^{\circ}, b_{6}=b_{10}=0$ ); (iv) the two-layer case with the same radial width, and with angular width of $\sim 35^{\circ}$ for the inner layer and $\sim 12.5^{\circ}$ for the outer one $\left(b_{6}=b_{10}=0\right)$.

\section{B. Evaluation of field gradient}

The computation of the field gradient for the sector layout is straightforward. For a sector of angular width $\alpha$, one has

$$
G \propto \int_{r}^{r+w} \int_{0}^{\alpha} \frac{\rho d \rho d \theta}{\rho^{2} e^{-2 i \theta}} \propto \sin (2 \alpha) \log \left(1+\frac{w}{r}\right)
$$

and therefore in the case of one layer made of sectors of width $w$, one has

$$
G=j \gamma_{0} \log \left(1+\frac{w}{r}\right)
$$

where the constant $\gamma_{0}$ depends on the layout: for the $\left[0^{\circ}-\right.$ $30^{\circ}$ ] sector, one has

$$
\gamma_{0}=10^{-6} \frac{4}{5} \sin 60^{\circ}=10^{-6} \frac{2}{5} \sqrt{3}
$$

i.e., $0.693 \times 10^{-6}[\mathrm{~T} \mathrm{~m} / \mathrm{A}]$ and for the $\left[0^{\circ}-24^{\circ}, 30^{\circ}-36^{\circ}\right]$ and for the $\left[0^{\circ}-18^{\circ}, 22^{\circ}-32^{\circ}\right]$ designs one finds, respectively,

$$
\gamma_{0}=10^{-6} \frac{4}{5}\left(\sin 72^{\circ}-\sin 60^{\circ}+\sin 48^{\circ}\right)
$$

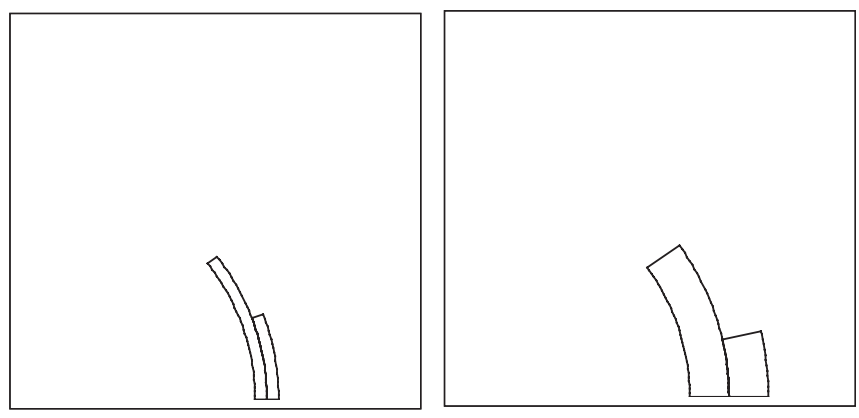

FIG. 9. Two shell layout solutions $\alpha_{1}=37^{\circ}, \alpha_{2}=20^{\circ}$ for $w / r=0.05$ (left) and $\alpha_{1}=35^{\circ}, \alpha_{2}=12.5^{\circ}$ for $w / r=0.16$ (right) that set $b_{6}$ and $b_{10}=0$. 


$$
\gamma_{0}=10^{-6} \frac{4}{5}\left(\sin 64^{\circ}-\sin 48^{\circ}+\sin 36^{\circ}\right)
$$

i.e., $0.663 \times 10^{-6}[\mathrm{Tm} / \mathrm{A}]$ and $0.634 \times 10^{-6}[\mathrm{Tm} / \mathrm{A}]$, respectively. For the two-layer case, one has

$$
\gamma(w, r)=\gamma_{1} \log \left(1+\frac{w}{r}\right)+\gamma_{2} \log \left(1+\frac{w}{r+w}\right),
$$

where $\gamma_{1}$ and $\gamma_{2}$ depend on the angular width of each layer.

\section{Evaluation of peak field}

The evaluation of the peak field is less straightforward than the previous case, and we propose to write it in the form

$$
\beta(r, w)=r \lambda(r, w) \gamma(r, w),
$$

where we have defined a dimensionless function $\lambda$, that is the ratio between the peak field and the gradient times the aperture. This parameter is equivalent to the ratio between the peak field in the coil and the main field in the center of the aperture of a dipole magnet. The dependence of $\lambda$ on $w$ for an aperture radius $r$ of $30 \mathrm{~mm}$ has been evaluated using a numerical computation (see Fig. 10). The shape of the curves is very similar in the four layouts: for increasing $w$, $\lambda$ initially decreases, then there is a minimum value, and then it increases linearly with $w$. One finds that $\lambda$ has a minimum, and that the value of the minimum is rather similar (between 1.13 and 1.16) in the analyzed cases. A good empirical fit of $\lambda$ is given by

$$
\lambda(r, w) \sim \lambda_{f}(r, w)=a_{-1} \frac{r}{w}+1+a_{1} \frac{w}{r}
$$

with $a_{-1}=0.06, a_{1}=0.10$ for the $\left[0^{\circ}, 30^{\circ}\right]$ design, and $a_{-1}=0.042, a_{1}=0.113$ for the $\left[0^{\circ}-24^{\circ}, 30^{\circ}-36^{\circ}\right] \mathrm{de}-$ sign (see Fig. 10).

The fact that, according to our numerical simulations, $\lambda$ and $\beta$ are linear in $w$ for large coil widths is not surprising: for the cross quadrupole we had a logarithmic increase stemming from

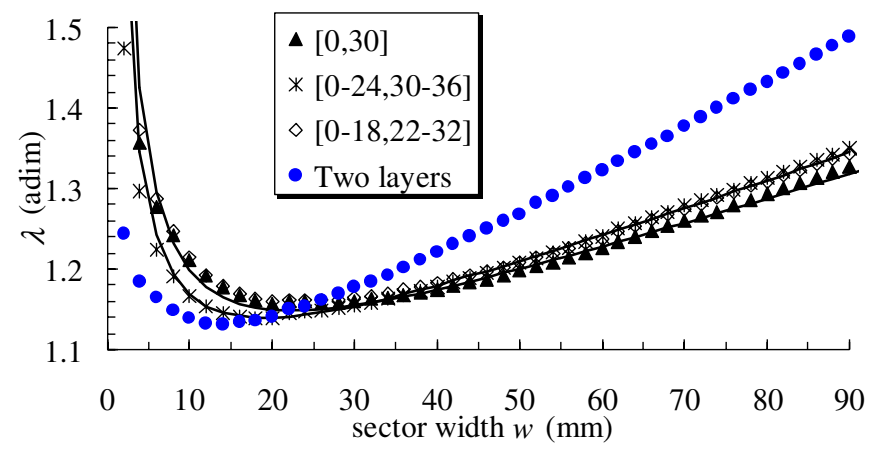

FIG. 10. (Color) Numerical evaluation of the function $\lambda$ defined in Eq. (31) versus sector width for different sector layouts, aperture radius of $30 \mathrm{~mm}$, and fitting curves as defined in (32) for the $0^{\circ}, 30^{\circ}$ and $0^{\circ}-24^{\circ}, 30^{\circ}-36^{\circ}$ design.

$$
\beta(r, w) \propto B_{p} \propto \int^{w} \frac{d \rho}{\rho}=\log (w),
$$

whereas in this case (sector coil) the integration is over a surface and therefore for $w \rightarrow \infty$

$$
\beta(r, w) \propto B_{p} \propto \int^{w} \frac{\rho d \rho}{\rho}=w .
$$

\section{Critical gradient versus conductor surface}

Using the expression (31) for $\beta$ in the equation defining the critical gradient, we obtain

$$
G_{c}=\frac{\kappa c B_{c 2}^{*}}{1+\kappa c \beta} \gamma=\frac{\kappa c B_{c 2}^{*}}{1+\kappa c r \gamma \lambda} \gamma
$$

Taking the limit for large width, $\gamma$ becomes large and therefore the critical gradient tends to

$$
G_{c} \rightarrow \frac{\kappa c B_{c 2}^{*}}{\kappa c r \gamma \lambda} \gamma=\frac{G_{c}^{*}}{\lambda}
$$

Since for the analyzed sector coils $\lambda$ linearly grows with $w$, we can expect that for $w \rightarrow \infty$ the critical gradient tends to zero, and therefore that there is an optimum value of the cable width that maximizes $G_{c}$, as in the cross-quadrupole case. Numerical simulations confirm this result (see Fig. 11): for an aperture radius $r=30 \mathrm{~mm}$, the maximum gradient is reached for a width of the sector which is around $50 \mathrm{~mm}$ for the one-layer case, and $30 \mathrm{~mm}$ for the two-layer case For larger widths one observes a slow decrease of $G_{c}$.

The first important result is that the maximum critical gradient is the same within $\pm 1 \%$ in the four analyzed layouts (see Fig. 11). The second result is also relevant: when the critical gradient is expressed as a function of the cross-sectional conductor surface, the curves relative to the four layouts agree within $\pm 1 \%$ (see Fig. 12). This allows us to conclude that for the analyzed cases the presence of a copper wedge, its angular position, and the presence of one or two layers does not affect the critical gradient that can be obtained with a given conductor surface. Therefore all these coil layouts are equivalent from an electromagnetic

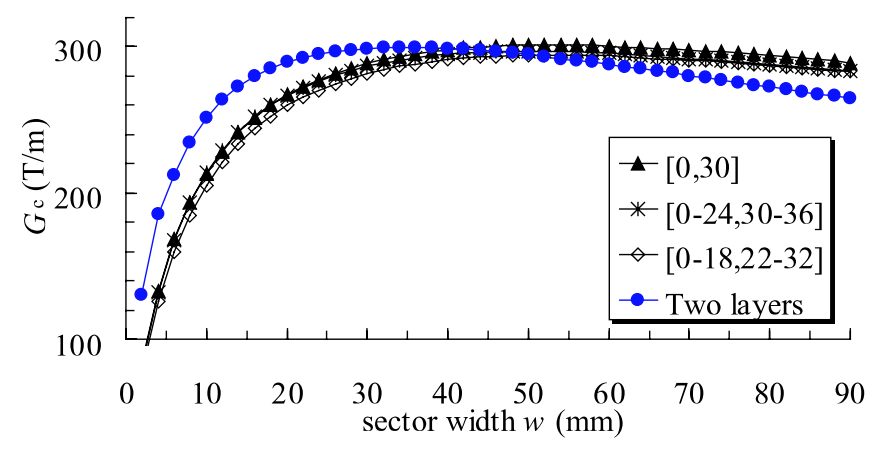

FIG. 11. (Color) Numerical evaluation of the critical gradient versus sector width for different sector layouts. 


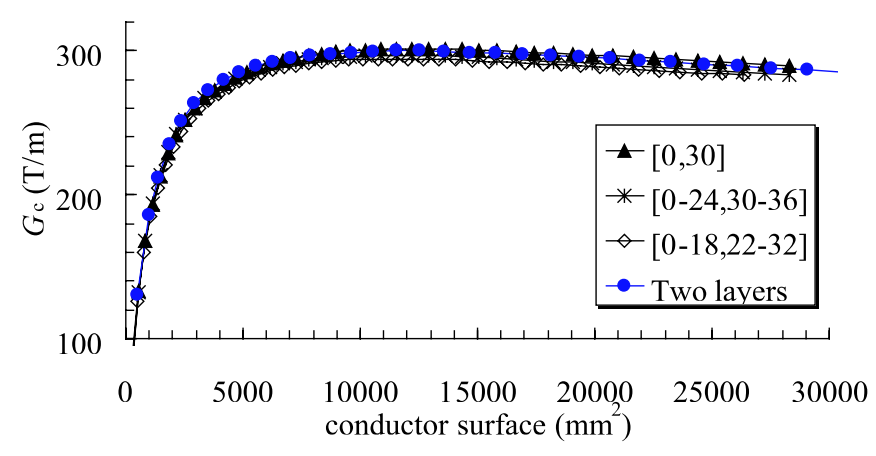

FIG. 12. (Color) Numerical evaluation of the critical gradient versus conductor surface for different sector layouts.

point of view. These are all radial sectors; in the next section we will show that on the other hand there is a dependence of the critical gradient on the inclination of the upper part of the coil, where the peak field is located.

\section{E. Analytical approximation}

We now propose an analytical approximation of the critical gradient as a function of the different parameters. We use Eq. (35), replacing $\gamma$ with its analytical expression (26), $\beta$ with (31), and we approximate $\lambda$ with the function $\lambda_{f}$, defined in (32), thus obtaining

$G_{c} \sim \frac{\kappa c B_{c 2}^{*} \gamma_{0} \log (1+w / r)}{1+\kappa c r\left[a_{-1}(r / w)+1+a_{1}(w / r)\right] \gamma_{0} \log (1+w / r)}$,

where we used the constants found for the $\left[0^{\circ}-24^{\circ}, 30^{\circ}-\right.$ $\left.36^{\circ}\right]$ case, i.e., $\gamma_{0}=0.663 \times 10^{-6}[\mathrm{Tm} / \mathrm{A}], \lambda_{f}(r, A)$ given by (32) and (38), with $a_{-1}=0.042, a_{1}=0.113$. We remind the reader that $w, r$ have to be expressed in $[\mathrm{m}], B$ in $[\mathrm{T}]$ and $c$ in $\left[\mathrm{A} / \mathrm{T} \mathrm{m}^{2}\right]$.

The comparison between the analytical expression (37) and the numerical result for the critical gradient is given in Fig. 13. The agreement is within $\pm 0.5 \%$ for $w / r>0.2$. For very thin coils, the approximation gets worse but still rather precise (for instance, $\pm 3 \%$ for $w / r=0.07$ ).

The previous equation can be also expressed in terms of the coil surface: for the $\left[0^{\circ}-24^{\circ}, 30^{\circ}-36^{\circ}\right]$ case, one has

$$
A=\frac{2 \pi}{3}\left[(r+w)^{2}-r^{2}\right]
$$

that gives

$$
1+\frac{w}{r}=\sqrt{1+\frac{3 A}{2 \pi r^{2}}}
$$

and therefore one can write

$$
G_{c} \sim \frac{\kappa c B_{c 2}^{*} \gamma_{0} \log \left[\sqrt{1+(3 A) /\left(2 \pi r^{2}\right)}\right]}{1+\kappa c r \lambda_{f}(r, A) \gamma_{0} \log \left[\sqrt{1+(3 A) /\left(2 \pi r^{2}\right)}\right]},
$$

where as in (37) $\gamma_{0}=0.663 \times 10^{-6}[\mathrm{Tm} / \mathrm{A}], \lambda_{f}(r, A)$ is

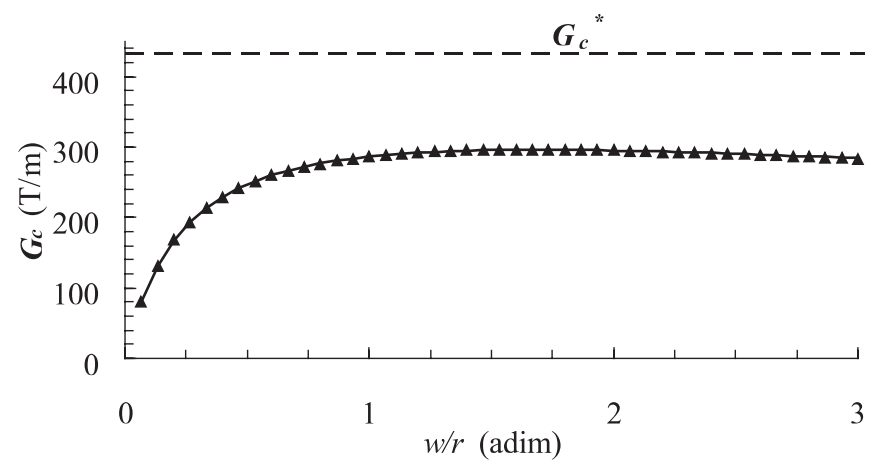

FIG. 13. Critical gradient versus sector width for the 0$24,30^{\circ}-36$ sector: numerical results (markers) and analytical approximation by (37) (solid line).

given by (32) and (38), with $a_{-1}=0.042, a_{1}=0.113, r$ has to be expressed in [m], $A$ in $\left[\mathrm{m}^{2}\right], B$ in [T], and $c$ in $\left[\mathrm{A} / \mathrm{T} \mathrm{m}^{2}\right]$.

Since the conductor area is a quantity which is not easy to appreciate, for a generic coil layout characterized by $A$ and $r$ we define an aspect ratio $w_{\text {eq }} / r$, where $w_{\text {eq }}$ is the width of a $\left[0^{\circ}-24^{\circ}, 30^{\circ}-36^{\circ}\right]\left(\right.$ or $\left.30^{\circ}\right)$ sector coil with the same area

$$
w_{\mathrm{eq}} \equiv\left(\sqrt{1+\frac{3 A}{2 \pi r^{2}}}-1\right) r
$$

and in the following sections we will express the results as a function of the aspect ratio $w_{\text {eq }} / r$ rather than in terms of coil surface $A$.

\section{F. Maximum critical gradient versus aperture radius}

Using the scaling law derived in (24), we extend the results of our numerical simulations made for a $30^{\circ}$ sector coil at $r=30 \mathrm{~mm}$ to different apertures. The maximum critical gradient divided by $G_{c}{ }^{*}$ is given in Table II and in Fig. 14 as a function of the aperture radius and of the dilution factor. We also explored dilution factors that are far from the usual accelerator magnets $(\kappa=0.25$ to 0.09$)$, but that can be relevant for the design of cables with internal cooling, and an extreme case of very low dilution $(\kappa=0.5)$.

One can make the following remarks.

(i) For an aperture radius of the order of $10 \mathrm{~mm}$ one can reach not more than $60 \%$ of $G_{c}{ }^{*}$. For $30 \mathrm{~mm}$ one obtains $\sim 75 \%$. For larger apertures, this fraction is increasing up to saturate at $\sim 85 \%$. This suggests that quadrupoles with a very small aperture do not manage to fully exploit the superconducting properties of the material.

(ii) An improvement of the dilution factor ( 0.4 instead of $0.25)$ gives a sizable increase of the maximum critical gradient only for apertures up to $30-40 \mathrm{~mm}(12 \%$ for $10 \mathrm{~mm}$ radius, $7 \%$ for $40 \mathrm{~mm}$ radius). 
TABLE II. Ratio between maximum critical gradient evaluated through simulations for a $\left[0^{\circ}-24^{\circ}, 30^{\circ}-36^{\circ}\right]$ sector coil and $G^{*}{ }_{c}$ given in Eq. (13).

\begin{tabular}{cccccccc}
\hline \hline \multicolumn{7}{c}{$r(\mathrm{~mm})$} \\
$k$ & 10 & 20 & 30 & 40 & 50 & 100 & 200 \\
\hline 0.50 & 0.60 & 0.69 & 0.74 & 0.76 & 0.78 & 0.81 & 0.84 \\
0.40 & 0.57 & 0.67 & 0.71 & 0.74 & 0.76 & 0.80 & 0.83 \\
0.33 & 0.54 & 0.64 & 0.69 & 0.72 & 0.74 & 0.79 & 0.83 \\
0.25 & 0.50 & 0.60 & 0.66 & 0.69 & 0.72 & 0.78 & 0.81 \\
0.17 & 0.43 & 0.54 & 0.60 & 0.64 & 0.67 & 0.74 & 0.79 \\
0.09 & 0.33 & 0.45 & 0.51 & 0.56 & 0.59 & 0.68 & 0.75 \\
\hline \hline
\end{tabular}

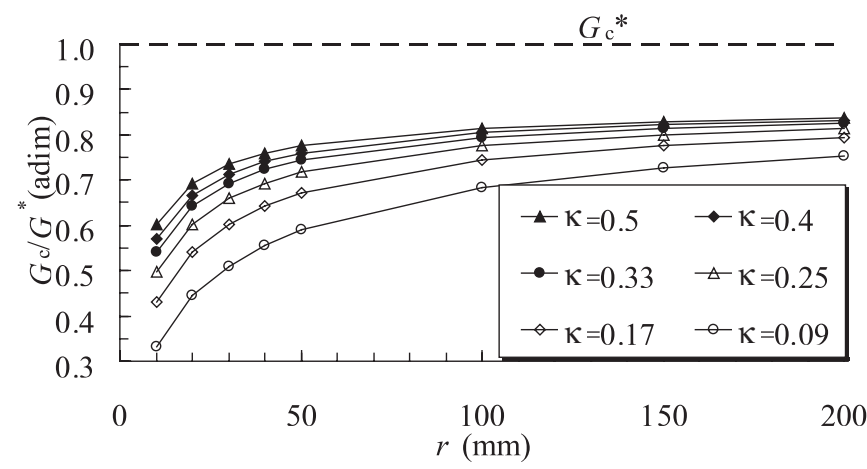

FIG. 14. Ratio between maximum critical gradient and $G^{*}{ }_{c}$ given in Eq. (13) versus aperture radius for a $\left[0^{\circ}-24^{\circ}, 30^{\circ}-36^{\circ}\right]$ sector coil.

Data of Figs. 11-13 show that the critical gradient has a very flat maximum: we therefore define an optimum cable width to get the highest gradient for a given aperture as the minimal width that provides $95 \%$ of the maximum critical gradient. Data are given in Fig. 15 and Table III.

(iii) For $\kappa=0.33$, this optimum cable width ranges from $1.7 r$ for an aperture of $10 \mathrm{~mm}$ to $r$ for $30 \mathrm{~mm}$, and $0.5 r$ for $200 \mathrm{~mm}$ : the larger the aperture, the lower the ratio $w / r$. For instance, for an aperture of $28 \mathrm{~mm}$ and $\kappa=0.33$, the optimum cable width is around $30 \mathrm{~mm}$, as used in the LHC main quadrupole (two layers of $15.4 \mathrm{~mm}$ cable width). This implies that the LHC main quadrupole is close to the maximum critical gradient within $5 \%$.

(iv) A smaller $\kappa$ requires larger widths to get the maximum gradient.

All these results depend on the slope $c$ of the critical surface, which is a property of the material but not of the temperature. Therefore, they are valid for $\mathrm{Nb}-\mathrm{Ti}$ both at 1.9 and at $4.2 \mathrm{~K}$. Different values for Tables II and III are obtained for materials with different slopes, or where the linear approximation is not valid, as in the $\mathrm{Nb}_{3} \mathrm{Sn}$ (see Sec. X).

\section{G. The one-layer layout without field quality}

We then consider a sector of angular width ranging from $10^{\circ}$ to $40^{\circ}$. In this case we neglect all aspects related to

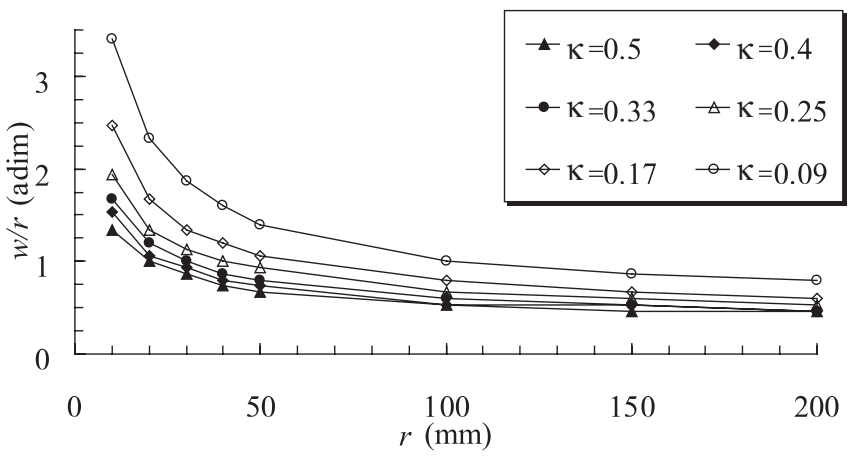

FIG. 15. Ratio between sector width and aperture radius that provides $95 \%$ of the maximum critical gradient versus aperture radius for a $\left[0^{\circ}-24^{\circ}, 30^{\circ}-36^{\circ}\right]$ sector coil.

TABLE III. Ratio between sector width and aperture radius that gives $95 \%$ of the maximum critical gradient for a $\left[0^{\circ}-\right.$ $24^{\circ}, 30^{\circ}-36^{\circ}$ ] sector coil.

\begin{tabular}{cccccccc}
\hline \hline \multicolumn{7}{c}{$r(\mathrm{~mm})$} \\
$k$ & 10 & 20 & 30 & 40 & 50 & 100 & 200 \\
\hline 0.50 & 1.33 & 1.00 & 0.87 & 0.73 & 0.67 & 0.53 & 0.47 \\
0.40 & 1.53 & 1.07 & 0.93 & 0.80 & 0.73 & 0.53 & 0.47 \\
0.33 & 1.67 & 1.20 & 1.00 & 0.87 & 0.80 & 0.60 & 0.47 \\
0.25 & 1.93 & 1.33 & 1.13 & 1.00 & 0.93 & 0.67 & 0.53 \\
0.17 & 2.47 & 1.67 & 1.33 & 1.20 & 1.07 & 0.80 & 0.60 \\
0.09 & 3.40 & 2.33 & 1.87 & 1.60 & 1.40 & 1.00 & 0.80 \\
\hline \hline
\end{tabular}

field quality, which will be not optimum except in the case of $30^{\circ}$ for $b_{6}$, i.e., we assume that the field harmonics can be compensated by corrector magnets. The aim of the simulation is to verify if relaxing the field quality constraint one can improve the maximum critical gradient.

The plot of the critical gradient versus the equivalent cable width defined in Eq. (41) shows that (see Fig. 16) for an aperture of $30 \mathrm{~mm}$ a sector from $25^{\circ}$ to $30^{\circ}$ is the optimum solution, the other sectors providing a smaller critical gradient for the same conductor surface.

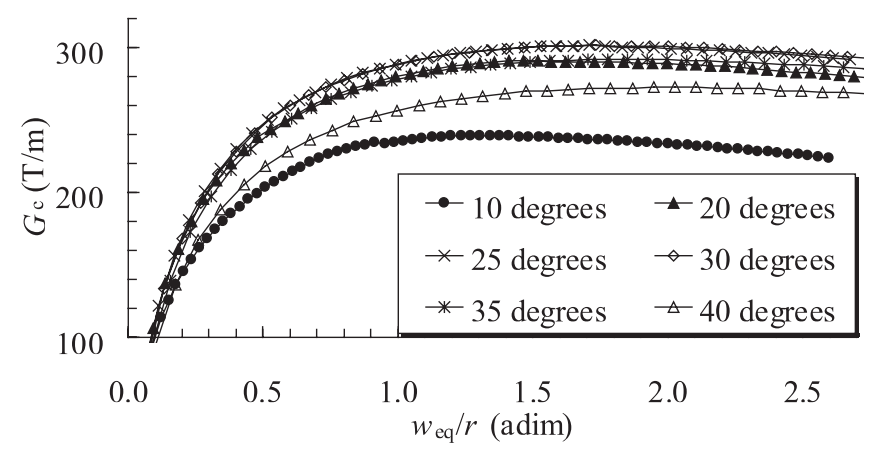

FIG. 16. Critical gradient versus equivalent sector width (41) for different angular widths of the sectors for an aperture radius of $30 \mathrm{~mm}$. 


\section{OTHER COIL LAYOUTS}

We also carried out simulations for other layouts to give some hints on other ways of arranging conductors. It must be pointed out that the manufacturing experience on these types of layouts is much less advanced with respect to the shell type, some cases being only academic. To simplify the analysis, we only partially satisfy the field quality constraint (here we have only $b_{6}=0$ ). We recall that in the shell case the further optimization of $b_{10}$ was not changing significantly the outcome of our analysis. Indeed, in all these layouts wedges can be always added to optimize also $b_{10}$, as for the shell case.

(i) A simplified open midplane design, given by a sector between $5^{\circ}$ and $25^{\circ}$. This layout preserves the condition $b_{6}=0$, and leaves $5^{\circ}$ of opening in the midplane.

(ii) A simplified block design [18], given by one rectangular block of rectangular conductors of width $w$ and height $h$, at a distance $r$ from the aperture center (see Fig. 17, left). The height of the block is set to have $b_{6}=0$.

(iii) A coil layout made of one block of rectangular conductors of width $w$, following the shell geometry, at a distance $r$ from the aperture center (see Fig. 17, right). We denote this layout by shell flattop. Also in this case, the height of the block is set to have $b_{6}=0$.

(iv) A coil layout made of one or more layers of a racetrack coil, of total width $w$, at a distance $r$ from the aperture center along the $45^{\circ}$ line (see Fig. 18, left). The number of cables in each layer is set to have $b_{6}=0$. This is a first approximation of the racetrack design, where wedges can be inserted to further optimize field quality $[20,21]$.

(v) A coil layout made of two intersecting ellipses, whose axes are $2 r$ and $2(r+w)$, respectively, thus giving in the magnet midplane an aperture radius of $r$ and a coil of width $w$ (see Fig. 18, right). The coil is filled with a uniform current density. This ideal case, well known in the literature [1-3], provides a pure quadrupole field, but has the main drawback that it cannot be wound from a large multistrand flat cable. We carry out the study of this rather academic case as it could be considered as the most effective layout. The peak field is on the intersection of the ellipses.

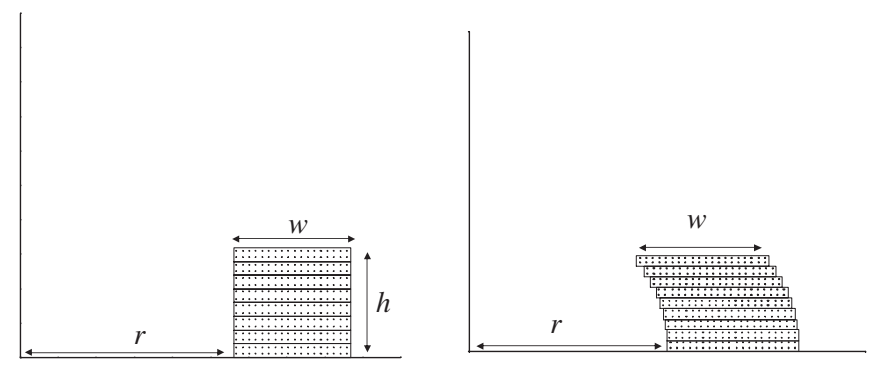

FIG. 17. The block (left) and the shell flattop (right) coil layouts (one eight of the whole cross section shown in the plot).
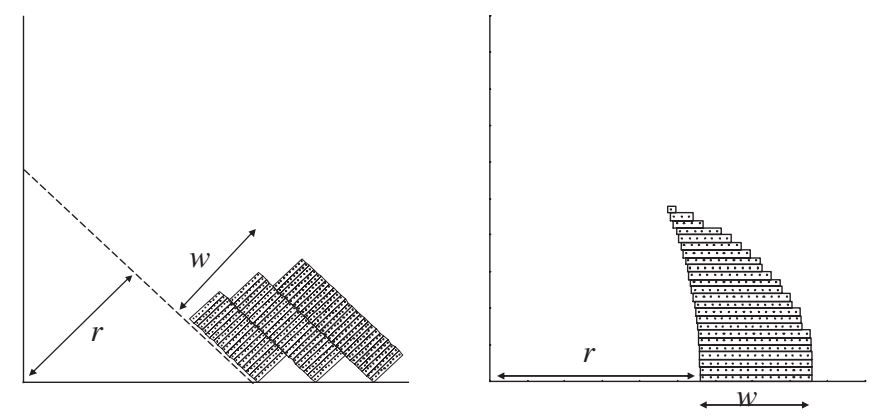

FIG. 18. The racetrack (left) and the intersecting ellipses (right) coil layouts (one eight of the whole cross section shown in the plot).

The estimate given in Eq. (26) for the parameter $\gamma$ is valid only for the open midplane design, that for the $\left[5^{\circ}, 25^{\circ}\right]$ case has a constant $\gamma_{0}$

$$
\gamma_{0}=10^{-6} \frac{4}{5}\left(\sin 50^{\circ}-\sin 10^{\circ}\right)
$$

i.e., $0.474 \times 10^{-6}[\mathrm{~T} \mathrm{~m} / \mathrm{A}]$. For the other types of design, we computed numerically the parameter $\gamma$ (always for our reference case of a $30 \mathrm{~mm}$ aperture radius). Results are given in Fig. 19 versus the aspect ratio defined in Eq. (41): one finds out that the field gradient per current density is very similar between the $30^{\circ}$ sector, the block coil, the shell flattop, and the intersecting ellipses layout, up to a coil width of $w=1.5 r$. The open midplane design at $\left[5^{\circ}, 25^{\circ}\right]$ and the racetrack provide $15 \%-20 \%$ less gradient than the first four cases.

For the parameter $\lambda$ (see Fig. 20), we find that the block layout has rather high values (between 1.3 and 1.4), while on the other hand the shell flattop has a very low value, always better than the $30^{\circ}$ sector, showing a much less significant increase with $w$. The effectiveness of this design could be due to the fact that the upper corner of the $30^{\circ}$ sector, where the peak field is usually located, is removed. This shows that, for realistic coils made up of conductor blocks, the inclination of the upper block can be a relevant parameter to get a higher critical gradient.

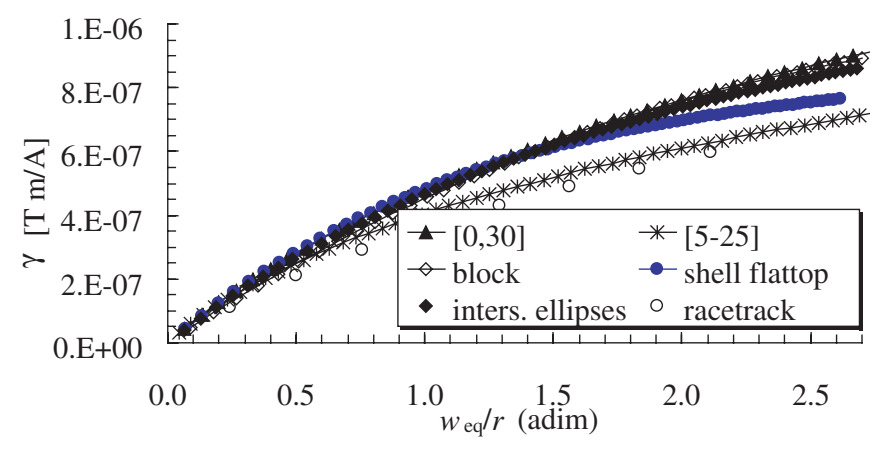

FIG. 19. (Color) Numerical evaluation of the parameter $\gamma$ versus aspect ratio (41) in four different coil layouts with an aperture radius of $30 \mathrm{~mm}$. 


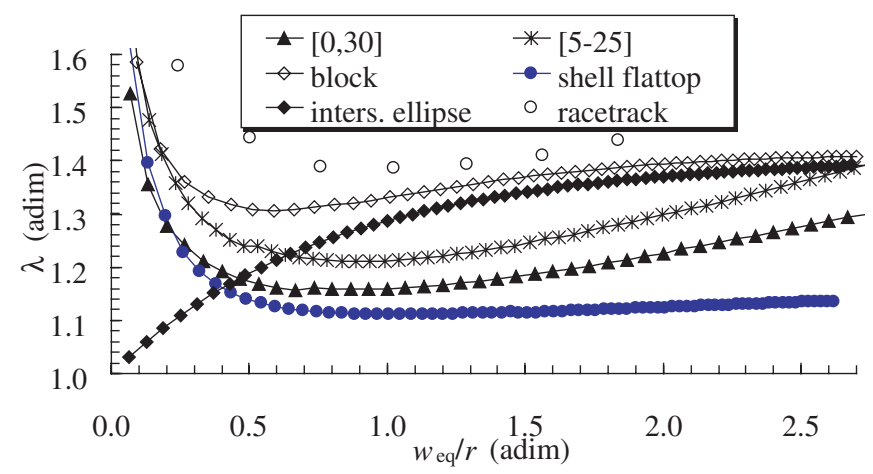

FIG. 20. (Color) Numerical evaluation of the parameter $\lambda$ versus equivalent sector width (41) in four different coil layouts with an aperture radius of $30 \mathrm{~mm}$.

For the intersecting ellipses, one can compute $\lambda$ as

$$
\lambda(r, w)=\frac{\sqrt{2}}{\sqrt{1+\frac{1}{[1+(w / r)]^{2}}}}
$$

and therefore for small $w$ the parameter $\lambda$ tends to one, since the aperture of the magnet becomes close to a circle, whereas for large $w$ it tends to 1.41 , since the aperture tends to a square. Therefore the gradient will saturate to its maximum value for increasing values of the coil width, and will not decay as for the sector coil.

Both for the block and for the shell flattop layouts the linear increase of $\lambda$ with $w$ is much less pronounced: indeed the argument used in Eqs. (33) and (34) is not valid any more since the height of the coil is not proportional to the width as in the sector case. Therefore, it remains an open question if these layouts feature a decrease of the critical gradient for very large coil widths, as for the sector case, or not, as for the intersecting ellipses. This issue is not relevant for practical purposes, since the coil sizes are extremely large. The racetrack design always features rather high values of $\lambda$.

Finally, we evaluate the critical gradient for the cases of an aperture of 10,30 , and $100 \mathrm{~mm}$ radius and $\kappa=0.28$

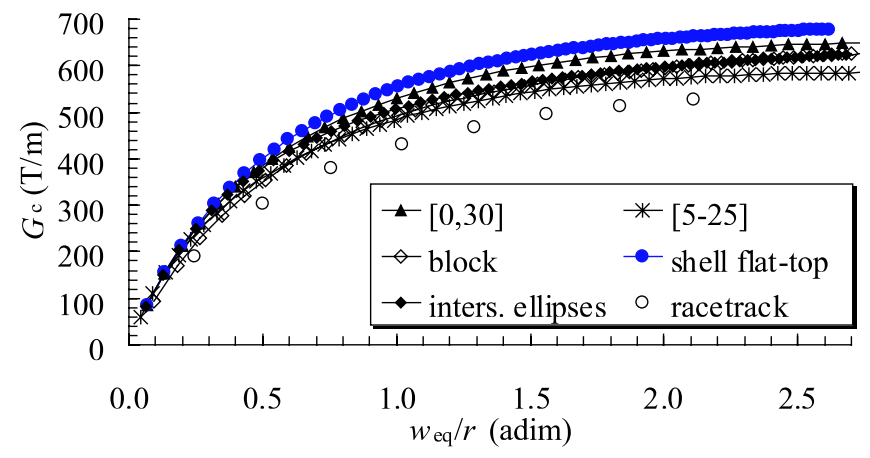

FIG. 21. (Color) Numerical evaluation of the critical gradient versus equivalent sector width (41) in six coil layouts with an aperture radius of $10 \mathrm{~mm}(\kappa=0.28)$.

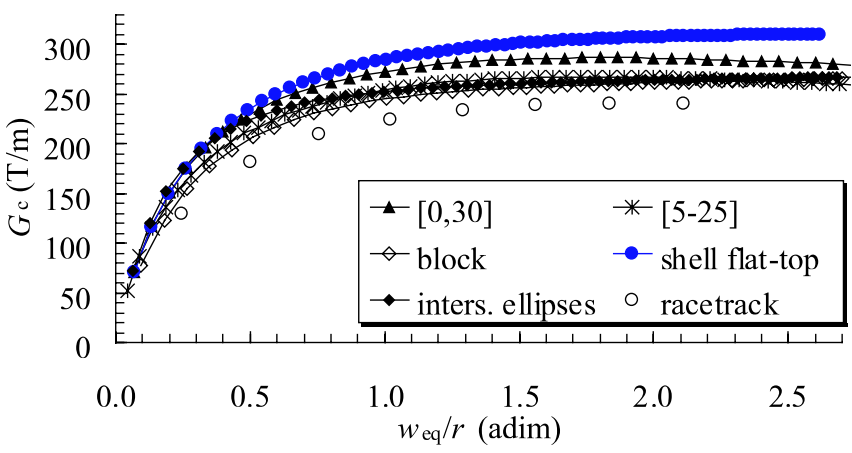

FIG. 22. (Color) Numerical evaluation of the critical gradient versus equivalent sector width (41) in six coil layouts with an aperture radius of $30 \mathrm{~mm}(\kappa=0.28)$.

(see Figs. 21-23). In order to be able to compare the different designs for the same coil surface (i.e., the quantity of superconductor), we always express our results in terms of the aspect ratio defined in (41). For the $30 \mathrm{~mm}$ case (Fig. 22) the shell flattop design provides at least $7 \%$ more in the maximum critical gradient, and is also more effective for small conductor areas. The $5^{\circ}$ open midplane gives $7 \%$ less than the $30^{\circ}$ sector. The block design gives $12 \%-13 \%$ less than the $30^{\circ}$ sector for the same amount of conductor, and the racetrack $15 \%$ to $20 \%$ less.

The differences are reduced for small apertures radii (see Fig. 21), and enhanced for large ones (see Fig. 23). The intersecting ellipses become more and more optimized for larger apertures. Indeed, this solution is far from being practical: therefore, one can state that for apertures ranging from 10 to $100 \mathrm{~mm}$ the shell layout (both the radial sector and even more the flattop) are well optimized, and that open midplane, block, and racetrack layouts are less effective by $5 \%-25 \%$.

For the racetrack case, that has a square aperture rather than a circular one, we consider the aperture radius as the radius of the inscribed circle (see Fig. 18, left). Indeed, a recent work [20] has shown that, for the case of the LHC upgrade, a shell layout of radius $r$ is equivalent to a racetrack layout of an aperture radius (inscribed circle) of

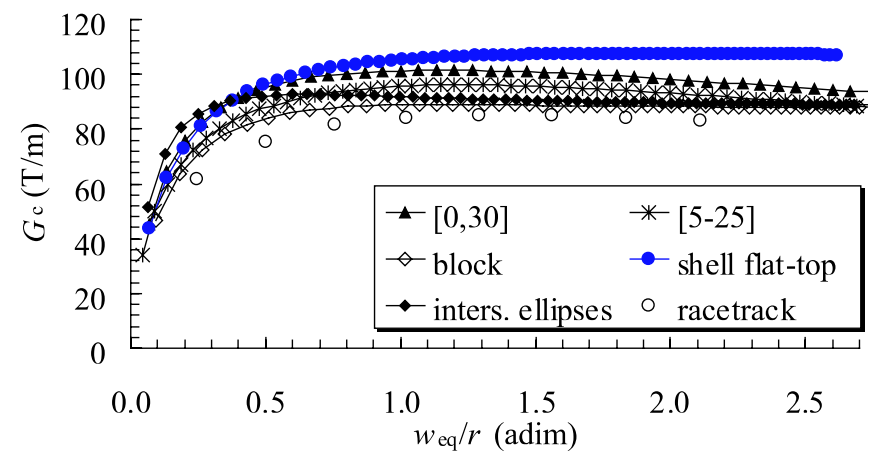

FIG. 23. (Color) Numerical evaluation of the critical gradient versus equivalent sector width (41) in six coil layouts with an aperture radius of $100 \mathrm{~mm}(\kappa=0.28)$. 
TABLE IV. Parameters of coil layouts of 13 superconducting quadrupoles.

\begin{tabular}{lccccc}
\hline \hline Name & $\begin{array}{c}\text { Ap. radius } \\
(\mathrm{mm})\end{array}$ & $\begin{array}{c}\text { Layers } \\
\text { (number, degrees) }\end{array}$ & Blocks & $\begin{array}{c}\text { Surface } \\
\left(\mathrm{mm}^{2}\right)\end{array}$ & $\begin{array}{c}\text { Aspect } \\
\text { ratio } w_{\text {eq }} / r\end{array}$ \\
\hline ISR MQ & 116 & $1[36]$ & 3 & 17725 & 0.28 \\
Tevatron MQ & 45 & $2[30,30]$ & $3[2,1]$ & 3385 & 0.35 \\
HERA MQ & 37 & $2[30,30]$ & $3[2,1]$ & 3542 & 0.49 \\
SSC MQ & 20 & $2[30,30]$ & $4[2,2]$ & 2274 & 0.92 \\
LEP I MQC & 90 & $1[32]$ & 2 & 11246 & 0.29 \\
LEP II MQC & 80 & $1[32]$ & 2 & 8184 & 0.27 \\
RHIC MQ & 40 & $1[30]$ & 2 & 1706 & 0.23 \\
RHIC MQY & 65 & $1[33]$ & 3 & 3411 & 0.18 \\
LHC MQ & 28 & $2[33,28]$ & $4[2,2]$ & 5013 & 1.01 \\
LHC MQM & 28 & $2[31,31]$ & $4[2,2]$ & 2593 & 0.61 \\
LHC MQY & 35 & $4[31,30,17,18]$ & $5[1,2,1,1]$ & 5674 & 0.79 \\
LHC MQXA & 35 & $4[33,30,20,20]$ & $6[2,2,1,1]$ & 8496 & 1.08 \\
LHC MQXB & 35 & $2[35,24]$ & $4[2,2]$ & 5395 & 0.76 \\
\hline \hline
\end{tabular}

around $15 \%$ less. Including this geometrical effect, the racetrack layout gives only 3\%-7\% less critical gradient with respect to the sector layout.

Please note that we always made the comparison of the critical gradient obtained by the different designs for the same area of conductor. If we compare the area of the conductor needed for getting the same critical gradient, the differences between the different layouts are greatly enhanced, due to the shape of the critical gradient vs aspect ratio curve. For instance, for the case of Fig. $22(r=$ $30 \mathrm{~mm}$ and $\kappa=0.28$ ), to get $250 \mathrm{~T} / \mathrm{m}$ one needs an aspect ratio of 0.67 for the sector coil, of 0.89 for an open midplane (i.e. $30 \%$ more of conductor), and 1.19 for a block coil (i.e. $\sim 80 \%$ more of conductor).

\section{ANALYSIS OF ACCELERATOR MAGNETS}

We finally compare the results of our analysis of simplified, uniform $j$ coil layouts with actual designs that have been used in accelerator magnets. In Table IV we give the main parameters of the geometry of 13 quadrupole designs that have been built in the past 30 years. For each one, we compute the aspect ratio $w_{\text {eq }} / r$ defined in Eq. (41). Apertures are ranging from 20 to $116 \mathrm{~mm}$, the aspect ratios from $1 / 6$ to 1 , and the conductor area spans over 1 order of magnitude. Designs are with one, two, or four layers, and 2 to 6 blocks. Each of the first ten quadrupoles are made with one type of cable, whereas the last three have different types of cables carrying the same current, thus leading to a different current density in the blocks. This technique, called current grading, aims at optimizing the coil design by making the best use of superconductor $[1,22,28,29]$.

The $\gamma$ of quadrupoles of Table IV, evaluated without iron, are plotted in Fig. 24 versus the aspect ratio $w_{\text {eq }} / r$ defined in Eq. (41): the remarkable result is that in the case of no current grading they all fit within $2 \%$ with the value computed for a $\left[0^{\circ}-24^{\circ}, 30^{\circ}-36^{\circ}\right]$ sector coil. This shows that for the analyzed cases (within 2\%), for a given quantity of cable one always obtains the same gradient per unit of current density, independently of the layer or sector subdivisions. For the cases with current grading, we used the current density of the sector where the peak field is located (usually the inner layer) to define $\gamma$ in Eq. (5). One can gain from $5 \%$ to $20 \%$ with respect to the $\left[0^{\circ}-\right.$ $24^{\circ}, 30^{\circ}-36^{\circ}$ ] sector coil.

Results for the parameter $\lambda$ are shown in Fig. 25, where we compare the actual values of the magnets given in Table IV without iron (markers) to the results for the $\left[0^{\circ}-24^{\circ}, 30^{\circ}-36^{\circ}\right]$ sector coil (solid line). The agreement is within $6 \%$. In particular, the magnet data confirm the trend that $\lambda$ increases for smaller aspect ratios $w_{\text {eq }} / r$. We also computed the case of a larger LHC quadrupole with two additional $30^{\circ}$ layers, giving a $w_{\text {eq }} / r=2.0$ : for this case $\lambda=1.22$, thus confirming the increase of $\lambda$ for large aspect ratios (solid line in Fig. 25). Magnets designed with a current grading have a lower $\lambda$ with respect to the sector estimate, but the LHC MQ is also very well optimized, reaching $\lambda=1.09$ without any current grading.

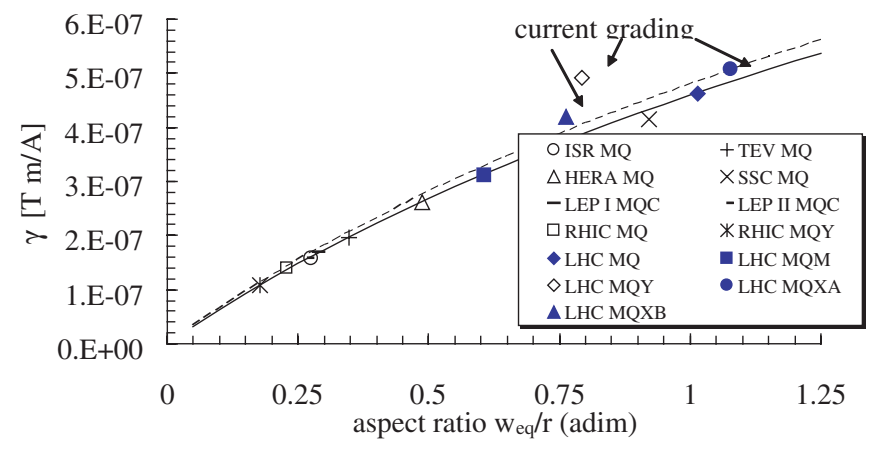

FIG. 24. (Color) Parameter $\gamma$ (field gradient per current density) for 13 accelerator quadrupoles without iron (markers) and results for a $30^{\circ}$ sector (dashed line) and $\left[0^{\circ}-24^{\circ}, 30^{\circ}-36^{\circ}\right]$ sector (solid line) versus aspect ratio as defined in Eq. (41). 


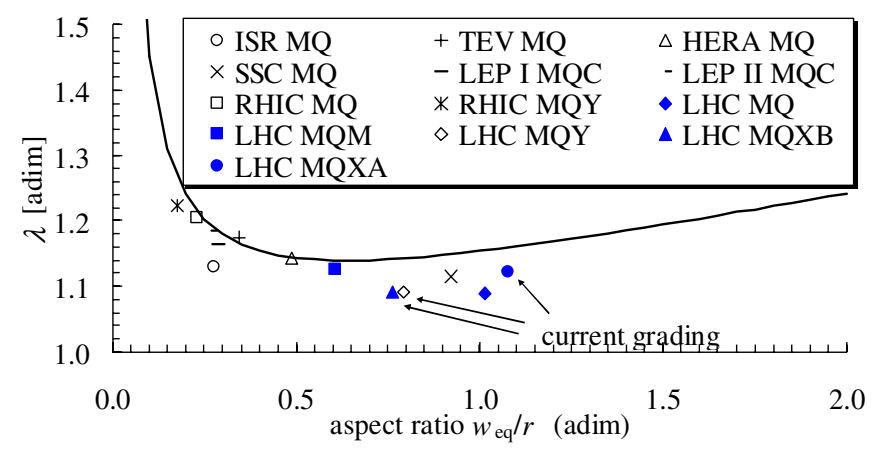

FIG. 25. (Color) Ratio $\lambda$ between peak field and current density vs aspect ratio as defined in Eq. (41): computation for a sector coil (solid line) and values for 13 quadrupoles evaluated without iron (markers).

Please note that, following Ref. [25], the values of built magnets are computed through a numerical code [30] that evaluates the field in a strand by considering the contribution of all the other strands, but neglecting the influence of the strand itself. This method underestimates the peak field of $1 \%--3 \%$, depending on the size of the strand. This partially justifies why most of the values of Fig. 25 are below the computed value for the sector coil.

In Table $\mathrm{V}$ we give a comparison between the estimated values for the critical gradient using the analytical approximation (40) with, i.e., $0.663 \times 10^{-6}[\mathrm{~T} \mathrm{~m} / \mathrm{A}], \lambda_{f}(r, A)$ given by (32) and (38), with $a_{-1}=0.042, a_{1}=0.113$, and the actual ones for the 13 analyzed magnets without iron. We assumed that same parameters for the $\mathrm{Nb}-\mathrm{Ti}$ critical surface at a given temperature as discussed in Sec. II C. The agreement in the case of no current grading is within $4 \%$. Magnets with current grading have a higher critical gradient with respect to our analytical benchmark of $3 \%$ to $9 \%$.

\section{IRON EFFECT}

The presence of iron has the main function of closing the magnetic circuit, i.e., shielding the external side of the magnet from the inner magnetic field. The iron also induces a higher field in the magnet aperture for the same current density, thus improving aspects related to protection. Indeed, it also induces a higher peak field and therefore the beneficial effect on the critical gradient is not the same in all cases. Finally, the iron yoke can also be used to withstand the forces (mechanical function). Here we will focus on its impact on the critical gradient. The field gradient increase due to the presence of a circular iron with constant permeability centered on the aperture at a distance $R_{i}$ can be analytically evaluated using well-known formulas [1]. Because of the structure of Eq. (10), the critical gradient increase due to a small variation of the coefficients $\gamma$ and $\beta$ is

$$
\frac{\Delta G_{c}}{G_{c}} \approx \frac{\Delta \gamma}{\gamma}-\frac{\Delta \beta}{\beta} \frac{\kappa c \beta}{1+\kappa c \beta} .
$$

If the relative increase of $\gamma$ and $\beta$ are the same, one has

$$
\frac{\Delta G_{c}}{G_{c}} \approx \frac{\Delta \gamma}{\gamma} \frac{1}{1+\kappa c \beta}
$$

and therefore, depending on the value of $\kappa c \beta$, the increase can be completely transferred to the critical gradient (current limited case, where $\kappa c \beta \ll 1$ ) or can leave the gradient unchanged (field limited case, where $\kappa c \beta \gg 1$ ).

Here, we computed the relative increase of $\gamma$ and $\beta$ for the coil layouts analyzed in the previous section. One finds that both coefficients increase by similar percentages, which range from 5\% to $20 \%$ for most cases, see Table VI. The magnets with lower aspect ratio (RHIC and ISR) have

TABLE V. Actual and analytical estimates of critical gradient for 13 superconducting quadrupoles (no iron).

\begin{tabular}{lccrrrr}
\hline \hline & \multicolumn{5}{c}{$\begin{array}{c}\text { Critical gradient } \\
\text { Actual }\end{array}$} \\
Analytical \\
Name & $\begin{array}{c}\text { Temperature } \\
(\mathrm{K})\end{array}$ & $\begin{array}{c}\kappa \\
\text { (adimensional) }\end{array}$ & $\begin{array}{c}\text { Relative error } \\
(\%)\end{array}$ & $\begin{array}{c}\text { Grading } \\
(\%)\end{array}$ \\
\hline ISR MQ & 4.4 & 0.35 & 61.9 & 60.2 & 2.8 & \\
Tevatron MQ & 4.4 & 0.25 & 115.4 & 117.0 & -1.4 & \\
HERA MQ & 4.4 & 0.27 & 151.2 & 152.1 & -0.6 & \\
SSC MQ & 4.4 & 0.27 & 268.9 & 268.9 & 0.0 & \\
LEP I MQC & 4.4 & 0.27 & 73.5 & 73.1 & 0.5 & \\
LEP II MQC & 4.4 & 0.33 & 79.0 & 79.4 & -0.4 & \\
RHIC MQ & 4.4 & 0.23 & 99.5 & 98.5 & 1.0 & \\
RHIC MQY & 4.4 & 0.27 & 73.2 & 72.2 & 1.3 & \\
LHC MQ & 1.9 & 0.25 & 289.2 & 278.9 & 3.7 & \\
LHC MQM & 1.9 & 0.26 & 248.7 & 248.4 & 0.1 & \\
LHC MQY & 4.4 & 0.34 & 207.8 & 190.7 & 9.0 & 43 \\
LHC MQXA & 1.9 & 0.34 & 266.0 & 257.6 & 3.2 & 10 \\
LHC MQXB & 1.9 & 0.33 & 258.3 & 243.7 & 6.0 & 27 \\
\hline \hline
\end{tabular}


TABLE VI. Increase of parameters $\gamma$ and $\beta$, and of the critical gradient, due to the iron yoke for some superconducting magnets.

\begin{tabular}{lccccccc}
\hline \hline & $\begin{array}{c}\text { Iron radius Collar thickness } \\
(\mathrm{mm})\end{array}$ & $(\mathrm{mm})$ & $(\%)$ & \multicolumn{1}{c}{$\Delta / \mathrm{B} / B$} & $\begin{array}{c}k c b \\
\text { (adimensional) }\end{array}$ & $\begin{array}{c}\Delta G_{c} \\
(\%)\end{array}$ & $\begin{array}{c}w_{\text {eq }} / r \\
\text { (adimensional) }\end{array}$ \\
\hline RHIC MQY & 90 & 13 & 39 & 32 & 1.4 & 16.6 & 0.18 \\
RHIC MQ & 55 & 5 & 45 & 38 & 0.9 & 22.6 & 0.23 \\
ISR MQ & 176 & 22 & 34 & 32 & 4.3 & 6.7 & 0.28 \\
Tevatron MQ & 101 & 41 & 8 & 8 & 1.5 & 2.6 & 0.35 \\
HERA MQ & 80 & 24 & 13 & 12 & 1.8 & 4.7 & 0.49 \\
LHC MQM & 102 & 27 & 7 & 7 & 1.5 & 2.7 & 0.61 \\
LHC MQXB & 92 & 26 & 10 & 11 & 3.1 & 2.1 & 0.76 \\
LHC MQY & 73 & 25 & 10 & 10 & 3.9 & 1.5 & 0.79 \\
SSC MQ & 60 & 20 & 7 & 7 & 1.5 & 2.4 & 0.92 \\
LHC MQ & 90 & 31 & 6 & 6 & 2.1 & 1.5 & 1.01 \\
LHC MQXA & 92 & 12 & 17 & 18 & 4.1 & 1.8 & 1.08 \\
\hline \hline
\end{tabular}

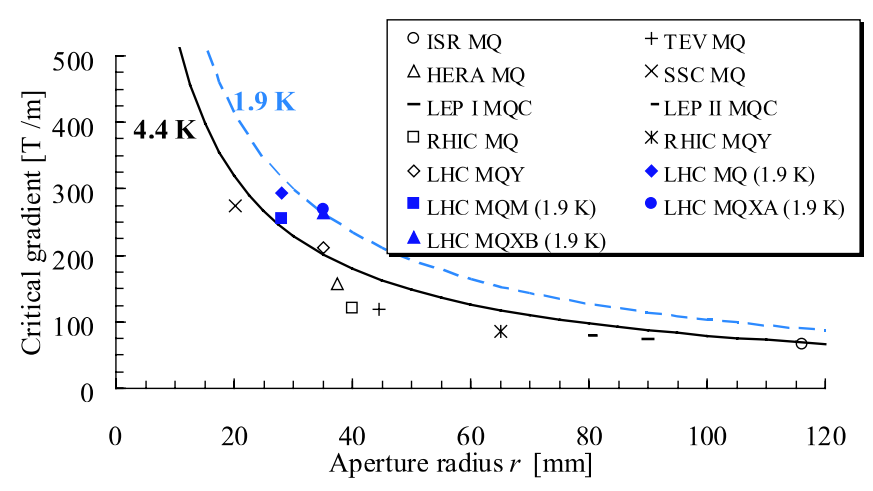

FIG. 26. (Color) Maximum critical gradient as a function of aperture radius for $\mathrm{Nb}-\mathrm{Ti}$ at 1.9 and $4.2 \mathrm{~K}$ for a sector coil of $\left[0^{\circ}-24^{\circ}, 30^{\circ}-36^{\circ}\right]$ (lines), and critical gradient in some superconducting quadrupoles (markers).

a much larger contribution of the iron to $\gamma$ and $\beta$ (32\% to $45 \%)$. One finds that for an aspect ratio larger than 0.5 the iron contribution is nearly negligible (1.5\% to $3 \%)$. Therefore we conclude that the iron does not affect the maximum critical gradient that can be reached for a given coil aperture.

A comparison between the actual critical gradients of the 13 analyzed quadrupoles (iron yoke included) to the estimate of the maximum critical gradient that can be obtained for a given aperture radius using the ironless $\left[0^{\circ}-24^{\circ}, 30^{\circ}-36^{\circ}\right]$ sector coil and a dilution factor of 0.33 is given in Fig. 26. All magnets are below our limit, with the exception of the MQY and MQXA that provide a few percent more $(2 \%-4 \%)$ than our estimate.

\section{AN APPLICATION TO THE LHC UPGRADE}

We finally apply the derived scaling laws to evaluate the case of a quadrupole $\mathrm{n} \mathrm{Nb}$-Ti at $1.9 \mathrm{~K}$ for the upgrade of the LHC insertion. For a $90 \mathrm{~mm}$ aperture $(r=45 \mathrm{~mm}), G^{*}{ }_{c}=$ $13 / 0.045=289 \mathrm{~T} / \mathrm{m}$. Using a cable with the most favorable dilution factor $(\kappa=0.33)$ we obtain (Table II) that we can reach at most $73 \%$ of $G^{*}$, i.e., $211 \mathrm{~T} / \mathrm{m}$. Table III shows that a coil width of $84 \%$ r i.e., $38 \mathrm{~mm}$, can give $95 \%$ of the last estimate, i.e., $201 \mathrm{~T} / \mathrm{m}$. Using a strong grading, one can expect to gain up to $10 \%$ to this estimate based on the sector coil with uniform current density, i.e., one can reach $221 \mathrm{~T} / \mathrm{m}$. Note that this estimate is rather far $(25 \%$ less) from the first naive guess $G^{*}{ }_{c}$. One has finally to subtract the margin needed for nominal operation (usually $20 \%$ to $30 \%$ ), that has been ignored in this analysis, thus giving 155 to $175 \mathrm{~T} / \mathrm{m}$. This agrees with the graded layout presented in [22].

For a $100 \mathrm{~mm}$ aperture, a simple scaling of the previous solution can be done, thus giving a critical gradient of $199 \mathrm{~T} / \mathrm{m}$ and an operational gradient of 139 to $159 \mathrm{~T} / \mathrm{m}$.

\section{AN ESTIMATE FOR $\mathrm{Nb}_{3} \mathrm{Sn}$}

$\mathrm{Nb}_{3} \mathrm{Sn}$ is a superconducting material characterized by a critical field $B_{c 2}^{*}$ in the range of $25 \mathrm{~T}$, i.e., around twice what is found for the Nb-Ti. The critical surface is not linear can be written in the Kramer form [31]

$$
j_{\mathrm{sc}}(B)=\frac{C(T, \varepsilon)}{\sqrt{B}}\left(1+\frac{B}{B_{c 2}^{*}(T, \varepsilon)}\right)^{2}
$$

where the two constants depend on temperature and strain. Using this equation with the parameters determined by what is considered at the moment the best available conductor (giving $3000 \mathrm{~A} / \mathrm{mm}^{2}$ at $12 \mathrm{~T}, 4.2 \mathrm{~K}$ ), we computed the maximum critical gradient of a sector coil versus the aperture radius. Results are shown in Fig. 27 for a filling ratio $\kappa=0.35$ at $1.9 \mathrm{~K}$ : one finds out that, due to the shape of the $\mathrm{Nb}_{3} \mathrm{Sn}$ critical surface (Fig. 28), the gain in the critical gradient with respect to $\mathrm{Nb}-\mathrm{Ti}$ is not $\sim 100 \%$ as one would expect from the increase of $B^{*}{ }_{c 2}$, but rather $\sim 50 \%$ for an aperture radius of $50 \mathrm{~mm}$. The gain improves for larger apertures. At $4.2 \mathrm{~K}$, the gain of $\mathrm{Nb}_{3} \mathrm{Sn}$ with respect to $\mathrm{Nb}-\mathrm{Ti}$ at $1.9 \mathrm{~K}$ is around $40 \%$ at $50 \mathrm{~mm}$. 


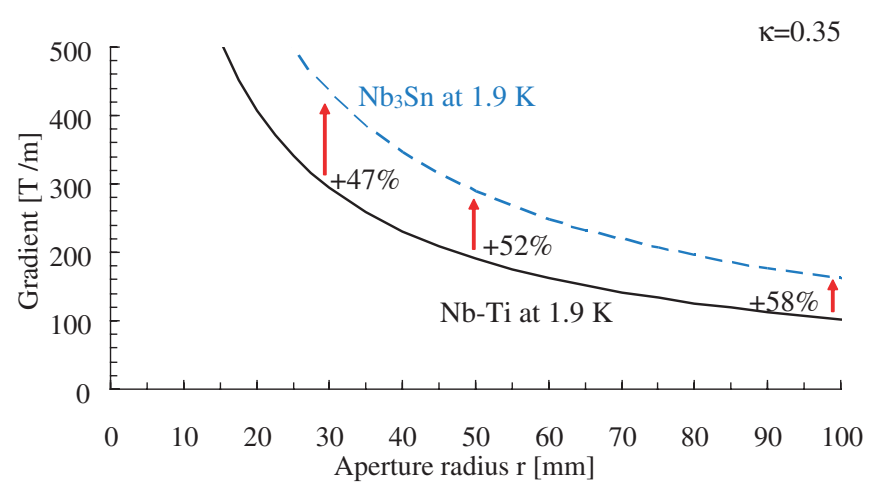

FIG. 27. (Color) Maximum critical gradient as a function of aperture radius for $\mathrm{Nb}-\mathrm{Ti}$ and $\mathrm{Nb}_{3} \mathrm{Sn}$ at $1.9 \mathrm{~K}$ for a sector coil of $\left(24^{\circ}, 30^{\circ}, 36^{\circ}\right), \kappa=0.35$.

An explicit equation for the critical gradient cannot be obtained from the Kramer form; indeed, a rather good analytic approximation can be derived using the following empirical fit of the critical surface

$$
j_{\mathrm{sc}}=c\left(1+\frac{b}{B}\right)
$$

This fit agrees within a few percent with (46) for typical parameters on a wide domain; for a ternary $\mathrm{Nb}_{3} \mathrm{Sn}$ giving $3000 \mathrm{~A} / \mathrm{mm}^{2}$ at $12 \mathrm{~T}, 4.2 \mathrm{~K}$, with a deformation of $\varepsilon=$ 0.003 , the agreement of (47) is within $5 \%$ from 5 to $15 \mathrm{~T}$ (with $c=3.9 \times 10^{9} \quad\left[\mathrm{~T} \mathrm{~m}^{2} / \mathrm{A}\right]$ and $b=21.0 \mathrm{~T}$, see Fig. 28). The empirical fit can be explicitly solved for the critical gradient

$$
G_{c}=\frac{\kappa c \gamma}{2}\left(\sqrt{\frac{4 b}{\lambda \gamma r \kappa c}+1}-1\right)
$$

where the parameters $\gamma$ and $\lambda$ have been defined in (26), (27), and (32).

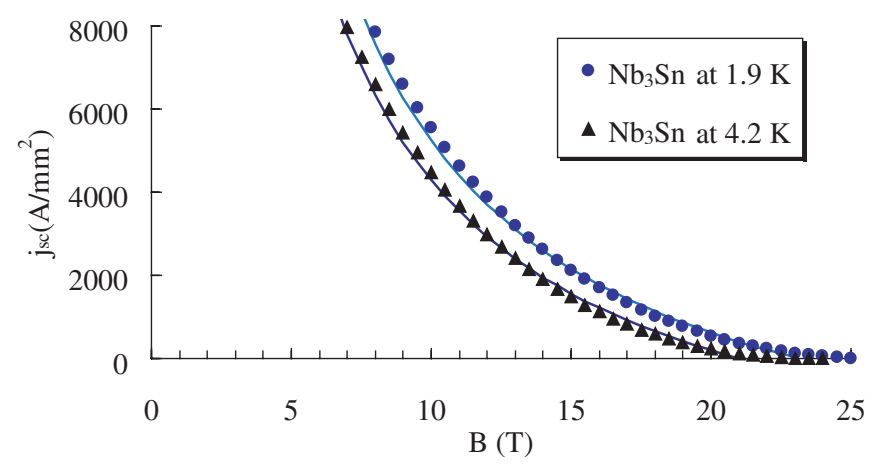

FIG. 28. (Color) Critical surface for $\mathrm{Nb}_{3} \mathrm{Sn}$ at $1.9 \mathrm{~K}$ and at $4.2 \mathrm{~K}$ according to Kramer law (markers), and fit given in Eq. (40) (solid line).

\section{SUMMARY}

We defined parameters that characterize the coil layout, namely, $\gamma$ (the field gradient per unit of current density) and $\beta$ (the peak field per unit of current density). Using a linear fit of the critical surface, we derived explicit expressions for the critical gradient (i.e., the gradient at the critical surface) in terms of the superconductor properties and of the coil layout parameters [see Eq. (10) and Sec. II].

Analysis of a simple model (the so-called cross quadrupole, Sec. III) where the coil layout parameters can be computed analytically revealed that there are three different regimes: in the current limited regime the critical gradient is linearly increasing with the coil width; it saturates in the intermediate regime, in which most of the magnet design are situated; finally, in the field limited regime it decreases. Therefore, there is an optimum coil size that provides the maximum critical gradient.

By inspecting the behavior of the critical gradient under rescaling of a generic layout, we found a scaling law [see Eq. (24) and Sec. IV] that allows one to extend the results for a given aperture radius to all apertures.

The analysis of the shell design (Sec. V) with radial sectors showed that one can explicitly compute $\gamma$ and one can define a fit for $\beta$ that agrees well with the numerical results in the range of interest for accelerator magnets. The main results are the following.

(i) One can write an explicit formula [see Eq. (40)] for the critical gradient as a function of the superconducting parameters, the layout parameters, and the geometric parameters (magnet aperture and conductor area).

(ii) The maximum critical gradient for aperture radii of $30 \mathrm{~mm}$ is $68 \%$ to $73 \%$ of the theoretical maximum gradient [defined as the ratio of the critical field in the superconductor $B^{*}{ }_{c 2}$ and the aperture radius, see Eq. (13)], for typical dilution factors $\kappa=0.3$ to 0.4 . This percentage depends strongly on the aperture radius, saturating at $\sim 85 \%$ for wider apertures, and reducing to $\sim 55 \%$ for an aperture radius of $10 \mathrm{~mm}$ (for $\kappa=0.3$ ). This suggests that quadrupoles with radial sector coil and very small apertures do not fully exploit the properties of the superconducting material. Moreover, the dependence on the dilution factor is more relevant for small apertures than for large apertures (see Table II). This result may lead to reconsider the design of conductor for the upgrade of the LHC IR quadrupoles, focused today on very low $\mathrm{Cu}$ content even for large apertures.

(iii) The decrease of the critical gradient for very large coils as found for the simple model is confirmed for the sector layouts. For a typical aperture radius of $30 \mathrm{~mm}$, and dilution factor $\kappa=0.33$, the $95 \%$ of the maximum critical gradient is reached by a radial width of the sector approximately equal to the aperture radius. For smaller apertures and for larger filing factors the optimum width is reached for larger $w / r$. A complete parametric analysis is given in Fig. 15 and Table III. 
A comparison of the different layouts has given the following results.

(i) The radial sector designs (both one layer and two layers, and with different positions of wedges) are equivalent in terms of maximum critical gradient to within $\pm 1 \%$, in the case of a constant $j$ in all sectors.

(ii) The radial sector designs provide very similar critical gradient (within $\pm 1 \%$ ) for the same conductor surface, and which fit well to the formula given in Eq. (40).

One can conclude that the radial sector coil layouts are equivalent in terms of critical gradient for a given quantity of superconductor. The selection of the coil layout (one layer, two layers, and angular position of the wedge) should be based on other considerations, such as the mechanical structure, stress management, the magnet protection, the choice of cable, and the aspects related to manufacturing. On the other hand, the inclination of the upper block, where the peak field is located, is a relevant parameter for reaching the highest critical gradient: the shell flattop analyzed in Sec. VI gives better results with respect to the radial sector cases.

The analysis of sketches of alternative coil layouts (Sec. VI) showed the following features for aperture radii of the order of $30 \mathrm{~mm}$.

(i) The arrangement of conductors in shell geometry with flattop (see Fig. 17, right) can give some improvement with respect to the shell radial sector layout ( $\sim 7 \%$ more).

(ii) A layout based on a rectangular block, whose sides are parallel or perpendicular to the midplane (see Fig. 18, left), is likely to be less effective than the shell radial sector coil ( $\sim 12 \%$ less $)$.

(iii) A design based on racetrack coil provides $15 \%$ to $20 \%$ less critical gradient (for the same conductor area).

(iv) An open midplane radial shell layout reduces the maximum critical gradient of $7 \%$ for a $5^{\circ}$ opening. This figure may be an acceptable fee to pay if a design has a primary goal to avoid heat deposition in the coils.

(v) The intersecting ellipse layout is not optimum. This is due to the fact that the aperture is not a circle, and for large coil widths it becomes like a square, putting it at a disadvantage with respect to the sector layout.

The analytical fit (40) to estimate the critical gradient was applied to 10 coil layouts used in accelerator magnets in the case of no iron contribution, showing an excellent agreement (within 4\%). Data relative to 3 magnets with current grading give a critical gradient that is $3 \%$ to $9 \%$ larger than our estimate based on no grading.

It was also confirmed that the impact of the iron on the critical gradient is negligible for magnets with a large aspect ratio $(w / r>0.5)$. We successfully checked the estimate of the maximum critical gradient based on the sector coil without iron to the actual values of 13 built magnets (iron included). We finally show that using that the best $\mathrm{Nb}_{3} \mathrm{Sn}$ conductor available at the moment one can obtain a 50\% improvement in the maximum critical gra- dient with respect to $\mathrm{Nb}-\mathrm{Ti}$ for apertures of the order of $50 \mathrm{~mm}$.

\section{CONCLUSION}

The performance of superconducting quadrupole magnets can be described approximately using analytic scaling laws. A set of such scaling laws has been derived and its validity verified by application to 13 existing quadrupoles that were built and successfully used in particle accelerators and storage rings.

\section{ACKNOWLEDGMENTS}

We wish to acknowledge all the colleagues that helped us in recovering the data of the magnet cross sections, and, in particular, B. Auchmann, L. Bottura, A. Devred, V. Kashikin, T. Nakamoto, S. Russenschuck, and T. Taylor. A special thanks to J.P. Koutchouk and T. Taylor for reading the manuscript and helpful comments.

\section{APPENDIX A: EQUATION FOR FIELD GRADIENT AND HARMONICS}

According to the complex formalism, a line carrying a current $I$ in the position $z_{0} \equiv x_{0}+i y_{0}$ gives a magnetic field $B(z) \equiv B_{y}(z)+\mathrm{i} B_{x}(z)$ in the position $z \equiv x+i y$ that reads

$$
B(z)=\frac{I \mu_{0}}{2 \pi\left(z-z_{0}\right)} .
$$

One can expand the series as

$$
\begin{aligned}
B(z) & =-\frac{I \mu_{0}}{2 \pi z_{0}} \sum_{n=1}^{\infty}\left(\frac{z}{z_{0}}\right)^{n-1} \\
& =-\frac{I \mu_{0}}{2 \pi z_{0}} \sum_{n=1}^{\infty}\left(\frac{R}{z_{0}}\right)^{n-1}\left(\frac{z}{R}\right)^{n-1},
\end{aligned}
$$

where $R$ is the reference radius, usually chosen as $2 / 3$ of the aperture radius. The multipolar expansion of the magnetic field according to the European notation $(n=1$ being the dipole) reads

$$
B(z)=\sum_{n=1}^{\infty} C_{n}\left(\frac{z}{R}\right)^{n-1}=\sum_{n=1}^{\infty}\left(B_{n}+i A_{n}\right)\left(\frac{z}{R}\right)^{n-1} .
$$

For a perfect quadrupole (with a fourfold symmetry) the first nonzero terms of the expansion are $B_{2}, B_{6}$, and $B_{10}$, and one can write the expansion as

$$
B(z)=B_{2} \frac{z}{R}+B_{6}\left(\frac{z}{R}\right)^{5}+B_{10}\left(\frac{z}{R}\right)^{9}+\cdots
$$

or in terms of the field gradient $G \equiv B_{2} / R$, expressed in $\mathrm{T} / \mathrm{m}$, and of the multipoles $b_{j} \equiv 10^{4} B_{j} / B_{2}$

$$
B(z)=G z\left[1+10^{-4}\left(b_{6} \frac{z^{4}}{R^{4}}+b_{10} \frac{z^{8}}{R^{8}}+\cdots\right)\right] .
$$


Please note that for accelerator superconducting magnets the multipoles must be of the order of 1 , and must be controlled within a fraction of a unit.

The first terms of (A2) are

$$
B(z)=-\frac{I \mu_{0}}{2 \pi\left(z_{0}\right)^{2}} z-\frac{I \mu_{0}}{2 \pi\left(z_{0}\right)^{6}} z^{5}-\frac{I \mu_{0}}{2 \pi\left(z_{0}\right)^{10}} z^{9}+\cdots
$$

and therefore comparing (A5) and (A6) for a current line at $z_{0}$ respecting the quadrupole symmetry (i.e., eight current lines), one has

$$
G=-8 \frac{I \mu_{0}}{2 \pi\left(z_{0}\right)^{2}}=-\frac{8}{5} \frac{I}{\left(z_{0}\right)^{2}} \times 10^{-6},
$$

where we substituted the actual value of $\mu_{0}=4 \pi \times 10^{-7}$ and we expressed $z_{0}$ in $\mathrm{m}$. The non-normalized multipoles read

$$
B_{n}=-16 \frac{R^{n-1}}{\left(z_{0}\right)^{n}} \times 10^{-7}
$$

\section{APPENDIX B: THE CROSS-QUADRUPOLE}

We consider a conductor of width $w$, thickness $t$ placed at a distance $r$ from the center of the aperture (see Fig. 5). The field gradient is given by

$$
G=-\frac{8}{5} j t \int_{r}^{r+w} \frac{d \rho}{\rho^{2}}=\frac{8}{5} t \frac{w}{r(r+w)} j .
$$

We first compute the field on the inner side of the horizontal conductor on the $x$ axis. Taking into account of the contribution of the four poles, one has

$$
B_{p}^{\text {in }}=\frac{\mu_{0} j t}{\pi}\left[\int_{0}^{w} \frac{d \rho}{\rho}-\int_{-2 r-w}^{-2 r} \frac{d \rho}{\rho}+2 r \int_{r}^{r+w} \frac{d \rho}{\rho^{2}+r^{2}}\right],
$$

where the first and the second integral are the contribution of the horizontal blocks on the positive and on the negative axis, respectively, and the third one is the contribution of both blocks on the $y$ axis. The first term has a logarithmic singularity in $\rho=0$, which is due to the fact that in our analytical approximation we consider a current density sheet of infinitely small thickness. This singularity disappears when a sheet of finite thickness is considered. We therefore write the integral as

$$
\int_{0}^{w} \frac{d \rho}{\rho}=\delta_{0}+\int_{w 0}^{w} \frac{d \rho}{\rho}=\delta_{0}+\log \left(\frac{w}{w_{0}}\right)
$$

where $w_{0}$ can be taken as the strand diameter and $\delta_{0}$ is the contribution of the single strand to the peak field on its surface. The other two integrals can be integrated analytically, thus giving

$$
B_{p}^{\text {in }}=\frac{\mu_{0} j t}{\pi} g^{\text {in }}(w, r),
$$

$$
\begin{aligned}
g^{\text {in }}= & \delta_{0}+\log \left(\frac{w}{w_{0}}\right)-\log \left(1+\frac{w}{2 r}\right)+2 \arctan \left(1+\frac{w}{r}\right) \\
& -\frac{\pi}{2} .
\end{aligned}
$$

Because of the different signs of the current in the horizontal and in the vertical blocks, the first and the third integral are positive, and the second is negative. The limit for infinite cable width shows that the first and the second contribution compensate each other, and therefore the peak field on the inner side of the conductor is limited by

$$
B_{p}^{\text {in }} p<\frac{\mu_{0} j t}{\pi}\left[\delta_{0}+\log \left(\frac{2 r}{w_{0}}\right)+\frac{\pi}{2}\right] .
$$

On the outer side of the conductor, the peak field can be computed as

$$
\begin{aligned}
B_{p}^{o u}= & \frac{\mu_{0} j t}{\pi}\left[-\int_{0}^{w} \frac{d \rho}{\rho}-\int_{2 r-2 w}^{-2 r-w} \frac{d \rho}{\rho}\right. \\
& \left.+2 r \int_{r}^{r+w} \frac{d \rho}{\rho^{2}+(r+w)^{2}}\right] .
\end{aligned}
$$

Here, the first and the second contribution have negative sign (now both blocks in the horizontal plane produce negative field), and the third is positive. Integration gives

$$
B_{p}^{\text {in }}=\frac{\mu_{0} j t}{\pi} g^{o u}(w, r)=\frac{\mu_{0} j t}{\pi}\left(-\delta_{0}+h^{o u}(w, r)-\frac{\pi}{4}\right),
$$

where

$$
\begin{aligned}
h^{\text {ou }}= & -\log \left(\frac{w}{w_{0}}\right)-\log \left(\frac{2 w+2 r}{w+2 r}\right) \\
& +\frac{2 r}{r+w} \arctan \left(1+\frac{w}{r}\right) .
\end{aligned}
$$

Here, for large $w$ the peak field is diverging as $\log (w)$ since we do not have the compensation between the horizontal blocks as before. Therefore, for large $w$ the peak field is on the outer side of the conductor. The functions $g^{\text {in }}(w, r)$ and $g^{o u}(w, r)$ are plotted versus $w$ for an aperture radius $r=$ $30 \mathrm{~mm}$ in Fig. 29. One observes that for moderate $w$, which is the domain of physical interest, the peak field is on the inner side of the conductor. For large values of the cable width (100 mm for our example of $r=30 \mathrm{~mm}$ ), the peak field in the inner side of the conductor starts to saturate, and the peak field in the outer side becomes larger.

The approximation of neglecting the contribution of the three blocks "far" from the spot where the peak field is evaluated, i.e.,

$$
B_{p}=\frac{\mu_{0} j t}{\pi}\left[\delta_{0}+\log \left(\frac{w}{w_{0}}\right)\right]
$$

shows to be rather good (within 10\% in the analyzed case). This approximation has the advantage of being sufficiently accurate, of having the correct behavior at infinity, and of 


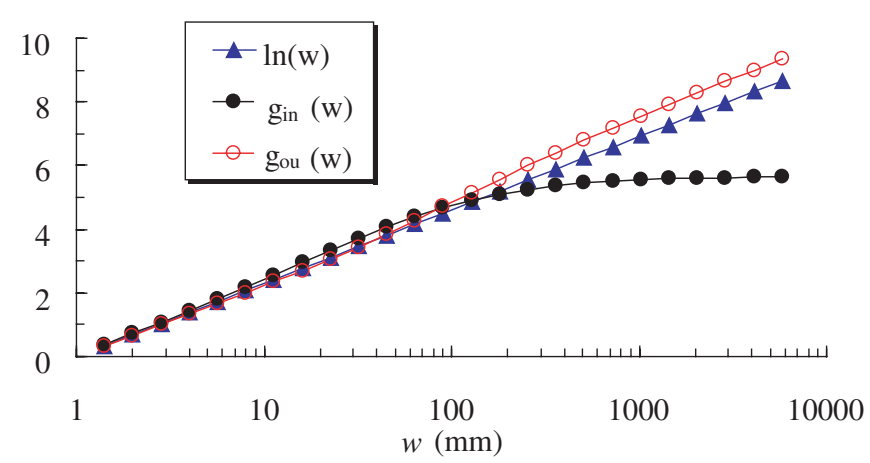

FIG. 29. (Color) Functions defined in (B5), (B8), and (B9) versus cable width $w$ for an aperture radius of $30 \mathrm{~mm}$.

improving the readability of the formulas. According to this approximation the peak field is independent of the aperture radius $r$.

\section{APPENDIX C: FIELD QUALITY CONSTRAINTS}

We consider a quadrupole whose coil layout (one fourth) is a sector of width $w$, from the angle $-\alpha$ to $\alpha$, at a distance $r$ from the center (see Fig. 2). The multipole coefficients can be obtained by integrating Eq. (A8) over the sector:

$$
B_{n}=\frac{4 \mu_{0} j R^{n-1}}{n \pi(2-n)}\left[\frac{1}{(r+w)^{n-2}}-\frac{1}{r^{n-2}}\right] \sin (n \alpha)
$$

For $\alpha=30^{\circ}$, the first order nonzero coefficient $B_{6}$ vanishes. Since the second order nonzero coefficient $B_{10}$ is proportional to $\sin (10 \alpha)$, it becomes zero for integer multiples of $18^{\circ}$. Therefore, a single radial sector of uniform $j$ cannot have $B_{6}=B_{10}=0$. If we consider a shell composed by two radial sectors $\left[0, \alpha_{1}\right]$ and $\left[\alpha_{2}, \alpha_{3}\right]$, i.e., we put a wedge between $\alpha_{1}$ and $\alpha_{2}$, the equations for setting $B_{6}=B_{10}=0$ are

$$
\begin{array}{r}
\sin \left(6 \alpha_{3}\right)-\sin \left(6 \alpha_{2}\right)+\sin \left(6 \alpha_{1}\right)=0, \\
\sin \left(10 \alpha_{3}\right)-\sin \left(10 \alpha_{2}\right)+\sin \left(10 \alpha_{1}\right)=0 .
\end{array}
$$

One can numerically compute the one-parameter family of solutions: $\alpha_{1}$ and $\alpha_{2}$, and the thickness of the wedge $\alpha_{2}-\alpha_{1}$ are shown versus $\alpha_{3}$ in Fig. 30. The minimal angular width of the wedge $\left(4^{\circ}\right)$ is at $\alpha_{3}=32^{\circ}$, and that it can go up to $10^{\circ}$ when the sector angle approaches the limiting and unphysical value of $\alpha_{3}=45^{\circ}$. There are four cases of solutions met for integer values of the angles, namely, $\left[0^{\circ}-12^{\circ}, 18^{\circ}-30^{\circ}\right], \quad\left[0^{\circ}-18^{\circ}, 22^{\circ}-32^{\circ}\right], \quad\left[0^{\circ}-24^{\circ}, 30^{\circ}-\right.$ $36^{\circ}$, and $\left[0^{\circ}-26^{\circ}, 36^{\circ}-44^{\circ}\right]$.

\section{Two-layer sector}

We now consider two circular shells of the same width, the first one from a distance $r$ to $r+w$, and the second from a distance $r+w$ to $r+2 w$. Each shell is composed

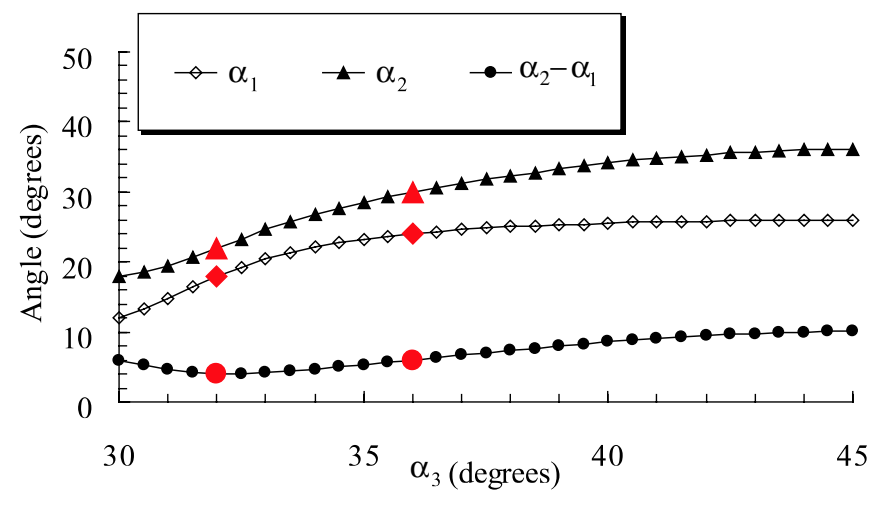

FIG. 30. (Color) One-parameter family for a single layer shell that sets $B_{6}$ and $B_{10}=0$. The two solutions shown in Fig. 7 are indicated by larger markers.

by one radial sector of angular width $\phi_{1}$ (inner layer) and $\phi_{2}$ (outer layer). We study if it is possible to set $B_{6}=$ $B_{10}=0$ by using the freedom in the choice of the angles $\phi_{1}$ and $\phi_{2}$. The equations read

$$
\begin{aligned}
B_{6}= & \lambda_{6}\left[\frac{1}{(r+w)^{4}}-\frac{1}{r^{4}}\right] \sin \left(6 \phi_{1}\right) \\
& +\lambda_{6}\left[\frac{1}{(r+2 w)^{4}}-\frac{1}{(r+w)^{4}}\right] \sin \left(6 \phi_{2}\right)=0 \\
B_{10}= & \lambda_{10}\left[\frac{1}{(r+w)^{8}}-\frac{1}{r^{8}}\right] \sin \left(10 \phi_{1}\right) \\
& +\lambda_{10}\left[\frac{1}{(r+2 w)^{8}}-\frac{1}{(r+w)^{8}}\right] \sin \left(10 \phi_{2}\right)=0 .
\end{aligned}
$$

For the case $w \ll r$, one has

$$
\begin{gathered}
\sin \left(6 \phi_{1}\right)+\sin \left(6 \phi_{2}\right)=0, \\
\sin \left(10 \phi_{1}\right)+\sin \left(10 \phi_{2}\right)=0,
\end{gathered}
$$

which has four solutions: $\left(3^{\circ}, 33^{\circ}\right),\left(21^{\circ}, 39^{\circ}\right),\left(33^{\circ}, 3^{\circ}\right)$, and $\left(39^{\circ}, 21^{\circ}\right)$. Among these solutions, the last is the more interesting since one has the larger sector in the inner layer.

Equations (C4) and (C5) are invariant under a rescaling of the aperture radius $r$ and the cable width $w$, i.e., a rescaling of the $(x, y)$ plane. Therefore, one can solve them for a given aperture $r$, and from the set of solutions $\phi_{1}(r, w) \phi_{2}(r, w)$ one can derive solutions for all apertures $R$ as $\alpha_{1}(R, R w / r) \alpha_{2}(R, R w / r)$. Using numerical methods, we compute the dependence of the two solutions $\left(33^{\circ}, 3^{\circ}\right)$ and $\left(39^{\circ}, 21^{\circ}\right)$ on $w / r$ (see Fig. 31). Both solutions exist only up to $w / r \sim 0.165$, where they collapse together. A good practical fit of the $\left(39^{\circ}, 21^{\circ}\right)$ is given by

$$
\phi_{1}=\eta_{2}\left(\frac{w}{r}\right)^{2}+\eta_{1} \frac{w}{r}+\eta_{0}
$$




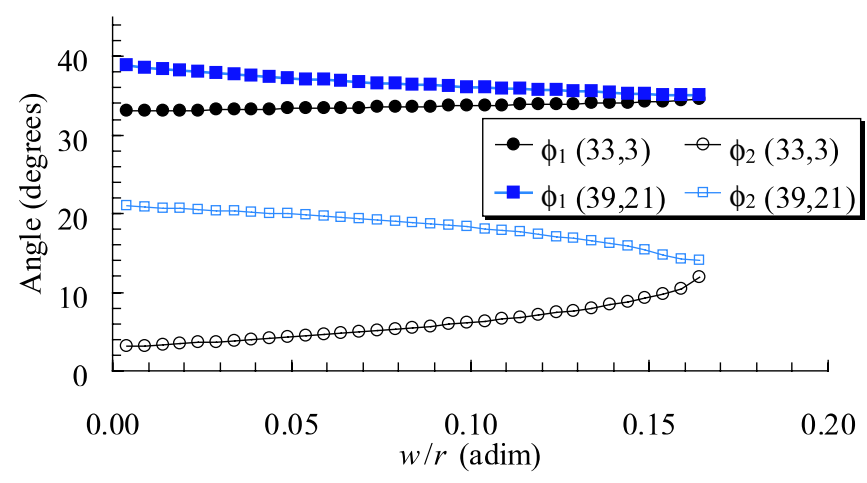

FIG. 31. (Color) Two solutions for setting to zero $B_{6}$ and $B_{10}$ : angular width of the inner and outer sector $\phi_{1}$ and $\phi_{2}$ versus ratio $w / r$.

with $\eta_{2}=62, \eta_{1}=-33$, and $\eta_{0}=39$, and

$$
\phi_{2}=\sqrt{\left(\kappa_{0}-\frac{w}{r}\right) \frac{1}{\kappa_{1}}}+\kappa_{2},
$$

with $\kappa_{2}=12.5, \kappa_{1}=0.0022$, and $\kappa_{0}=0.165$. Indeed, the maximum ratio $w / r$ allowed for the existence of the solution is very small $(1 / 6)$ and usually is not met. For $w / r$ larger than $1 / 6$, one must add a wedge in the inner sector, thus setting $B_{6}=B_{10}=0$. This layout has been adopted in several magnet designs (for instance, the LHC insertion quadrupoles MQY and MQXA).

[1] H. Brechna, Superconducting Magnet Systems (SpringerVerlag, Berlin, 1973).

[2] M.N. Wilson, Superconducting Magnets (Clarendon Press, Oxford, 1983).

[3] K. H. Mess, P. Schmuser, and S. Wolff, Superconducting Accelerator Magnets (World Scientific, Singapore, 1996).

[4] J. Billan et al., CERN Yellow Report 76-16, 1976.

[5] W. E. Cooper et al., Fermilab Report TM-1183, 1983.

[6] S. Wolff, The Superconducting Magnet System for HERA, edited by C. Marinucci and P. Waymuth (SIN, Zurich, 1995).

[7] P. J. Ferry et al., CERN LEP-MA 89 (1989).

[8] T. Taylor et al., CERN AT-MA 91, 1991.

[9] E. Bleser et al., Nucl. Instrum. Methods Phys. Res., Sect. A 235, 435 (1985).
[10] R. Kreutz, "Design Considerations on the SSC Collider Quadrupole Magnets," in the European Particle Accelerator Conference (1992), pp. 1423-1425.

[11] M. Anerella et al., Nucl. Instrum. Methods Phys. Res., Sect. A 499, 280 (2003).

[12] AA. VV., LHC Design Report, CERN 2004-003 (2004).

[13] T. Sen et al., "Beam physics issues for a possible 2nd generation LHC IR," in the European Particle Accelerator Conference (2002), pp. 371-373.

[14] J. Strait et al., "Towards a New LHC Interaction Region Design for a Luminosity Upgrade," in the Particle Accelerator Conference (2003), pp. 42-45.

[15] F. Ruggiero et al., "Performance Limits and IR Design of a Possible LHC Luminosity Upgrade Based on Nb-Ti SC Magnet Technology," in the European Particle Accelerator Conference (2004), pp. 608-610.

[16] G. Loew, "Report from the International Linear Collider Technical Review Committee," in the Particle Accelerator Conference (2003), pp. 647-651.

[17] V. Kashikhin and A. Zlobin, IEEE Trans. Appl. Supercond. 12, 107 (2002).

[18] P. Ferracin et al., "Field Quality Analysis of the Next Generation IR Quadrupole for the LHC," in the Particle Accelerator Conference (2003), pp. 1984-1986.

[19] A. Zlobin et al., "Aperture Limitations for 2nd Generation $\mathrm{Nb}_{3}$ Sn LHC IR Quadrupoles," in the Particle Accelerator Conference (2003), pp. 1975-1977.

[20] V. Kashikhin et al., "2nd generation LHC IR Quadrupoles Based on $\mathrm{Nb}_{3} \mathrm{Sn}$ Racetrack Coils," in the European Particle Accelerator Conference (2004), pp. 1774-1776.

[21] P. Ferracin et al., IEEE Trans. Appl. Supercond. 15, 1132 (2005).

[22] R. Ostojic et al., "Low- $\beta$ Quadrupole Designs for the LHC Luminosity Upgrade," in the Particle Accelerator Conference (2005), pp. 2795-2797.

[23] A. Tollestrup, Fermilab UPC-086 (1979).

[24] Vector fields, http://www.vectorfields.com

[25] CERN Yellow Report 99-01, 1999), edited by S. Russenschuck.

[26] L. Rossi, IEEE Trans. Appl. Supercond. 12, 219 (2002).

[27] S. Caspi and P. Ferracin, "Limits of $\mathrm{Nb}_{3} \mathrm{Sn}$ Accelerator Magnets," in the Particle Accelerator Conference (2005), pp. 107-111.

[28] S. Caspi, P. Ferracin, and S. Gourlay, in the 19th Magnet Technology Conference [IEEE Trans. Appl. Supercond. (to be published)].

[29] G. Ambrosio et al., INFN TC-95-25, 1995.

[30] E. Todesco, Bio' code (unpublished).

[31] E. J. Kramer, J. Appl. Phys. 44, 1360 (1973). 